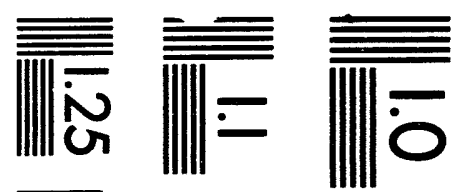

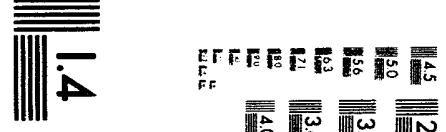

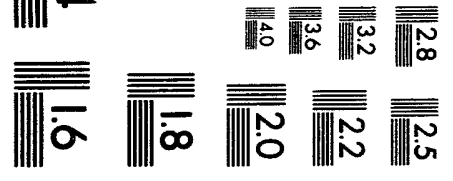


LBL-34460

UC-800

\title{
Design Calculations for a \\ Combined Ventilation and Brine Injection Experiment at the Grimsel Rock Laboratory, Switzerland
}

\author{
Stefan Finsterle and Karsten Pruess \\ Earth Sciences Division \\ Lawrence Berkeley Laboratory \\ University of California \\ Berkeley, CA 94720
}

July 1993

This work was carried out under U.S. Department of Energy Contract No. DE-AC03-76SF00098 for the Director, Office of Civilian Radioactive Waste Management, Office of External Relations, and was administered by the Nevada Operations Office, U.S. Department of Energy, in cooperation with the Swiss National Cooperative for the Disposal of Radioactive Waste (Nagra).

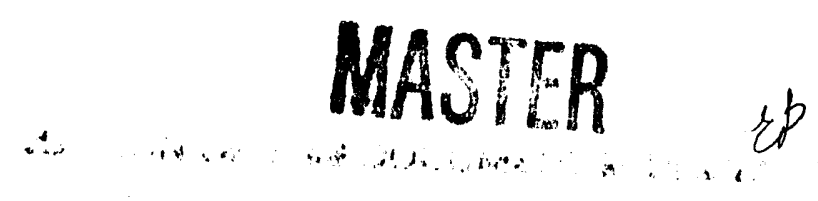




\begin{abstract}
A combined ventilation and brine injection test is planned at the Grimsel Test Site. The objective of the experiment is to study the transport of liquid and gas in the vicinity of a ventilated drift in orcer to evaluate the impact of the drying process on the characterization of the rock matrix. The propnsed test sequence includes a desaturation - resaturation cycle. In addition, brine and fresh water will be injected from a borehole as trace electrolytes in order to better track the propagation of the individual phases. Results of design calculations using the TOUGH 2 code show that injection of brine may significantly influence the unsaturated flow behavior by changing the pressure and saturation distribution around the borehole. Transport velocity is predicted to be very slow, requiring several months for the brine to reach the drift wall. However, the presence of preferential flow paths may reduce travel time and alter brine content and saturation distribution so that certain sensors may respond earlier or not at all.
\end{abstract}




\section{Tablc of Contents}

page

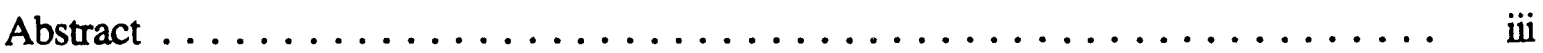

Table of Contents $\ldots \ldots \ldots \ldots \ldots \ldots \ldots \ldots \ldots \ldots \ldots \ldots \ldots \ldots \ldots \ldots$

List of Figures $\ldots \ldots \ldots \ldots \ldots \ldots \ldots \ldots \ldots \ldots \ldots \ldots \ldots$

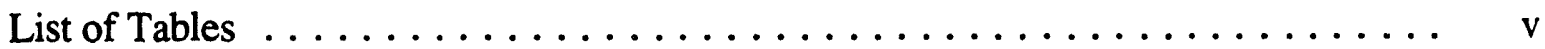

1. Background and scope $\ldots \ldots \ldots \ldots \ldots \ldots \ldots \ldots \ldots \ldots \ldots \ldots \ldots$

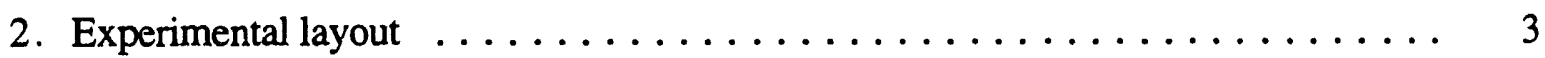

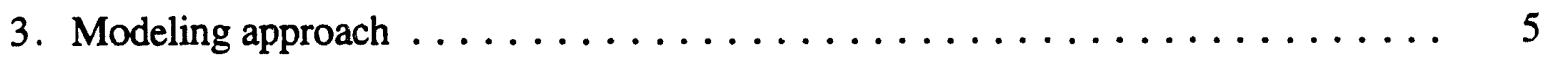

3.1 Fluid properties and physical processes $\ldots \ldots \ldots \ldots \ldots \ldots$

3.2 Moisture transfer at the drift surface $\ldots \ldots \ldots \ldots \ldots \ldots \ldots$

3.3 Model domain and computational grid $\ldots \ldots \ldots \ldots \ldots \ldots \ldots \ldots$

3.4 Formation parameters, initial and boundary conditions . . . . . . . . 11

3.5 Simulation results for homogeneous medium $\ldots \ldots \ldots \ldots \ldots \ldots$

3.6 Simulation results for heterogeneous medium $\ldots \ldots \ldots \ldots \ldots \ldots$

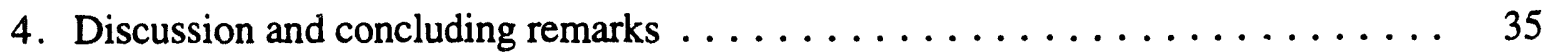

5. Acknowledgment $\ldots \ldots \ldots \ldots \ldots \ldots \ldots \ldots \ldots \ldots \ldots \ldots \ldots \ldots \ldots$

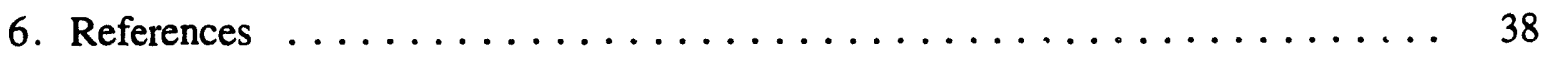




\section{List of Figures}

page

Figure 1: Schematic of ventilation drift, borehole, and equipment $\ldots \ldots \ldots \ldots$

Figure 2: Schematic of moisture transfer at the drift wall $\ldots \ldots \ldots \ldots$

Figure 3: Model domain and computational grid $\quad \ldots \ldots \ldots \ldots \ldots$

Figure 4: Comparison between measured (symbols) and calculated (solid lines) . . . 13 water potentials during desaturation experiment

Figure 5: Characteristic curves determined from inverse modeling of desaturation $\ldots 13$ experiment

Figure 6: Brine content and gas saturation, homogeneous medium $\ldots \ldots \ldots$. . . 19

Figure 7: Gas saturation profiles during resaturation $(\operatorname{Step} 5 \mathrm{~A}) \ldots \ldots 22$

Figure 8: Relative brine content and liquid saturation at sensor TDR1.60 _ . . . 23

Figure 9: Water potential at sensor TP1.30, homogeneous medium . . . . . . . 24

Figure 10: Brine content and gas saturation, heterogeneous medium $\ldots \ldots \ldots 28$

Figure 11: Brine content profiles at the end of test sequence $A \ldots \ldots 29$

Figure 12: Brine content profiles at the end of test sequence $B \ldots \ldots$

Figure 13: Brine content at TDR sensors, test sequence A, heterogeneous medium _ . 31

Figure 14: Brine content at TDR sensors, test sequence B, heterogeneous medium $\quad \ldots \quad 32$

Figure 15: Water potential at TP sensors, test sequence A, heterogeneous medium $\quad \ldots \quad 33$

Figure 16: Water potential at TP sensors, test sequence B, heterogeneous medium . 34

\section{List of Tables}

page

Table 1: $\quad$ Proposed test sequence $\ldots \ldots \ldots \ldots \ldots \ldots \ldots \ldots \ldots \ldots \ldots \ldots \ldots$

Table 2: Formation parameters, initial and boundary conditions $\ldots \ldots \ldots \ldots$

Table 3: Variances, covariances, and correlation coefficients $\ldots \ldots \ldots \ldots$ 


\section{Background and Scope}

Ventilation tests in mined caverns were originally conceived to determine the macropermeability of tight rocks by measuring the inflow into drift sections with controlled ventilation [Wilson et al., 1981; Vomvoris \& Frieg, 1991]. The customary interpretation of such tests views the ventilation air simply as a convenient means to convey the incoming moisture to a measuring device. Accordingly, ventilation test interpretation is based on the assumption that flow toward the drift is single-phase. However, the permeability of the granodiorite matrix may be affected by partial drying out of the drift wall leading to substantial regions with two-phase flow conditions and negative water potentials. As a consequence, the transport processes in the vicinity of the drift may be influenced by twophase flow effects.

A joint project between the Institute of Terrestrial Ecology, ETH Zürich, and the Swiss National Cooperative for the Disposal of Radioactive Waste (Nagra) has been initiated to quantify the extent of the two-phase region and study its hydraulic properties. Laboratory and in-situ measurements of water potential, water content, temperature, and ambient air humidity were performed at the Grimsel Test Site (GTS) [Schneebeli et al., 1990]. The direct measurement of water potentials with Thermocouple Psychrometers (TP) during the 1991 resaturation experiment established that a two-phase zone extended up to $1.6 \mathrm{~m}$ from the drift wall into the rock [Schneebeli et al., 1992]. The propagation of the drying front was observed during a six month ventilation period starting November 26, 1991 [Gimmi et al., 1992]. Water content measurements using Time Domain Reflectometry (TDR) turned out to be difficult mainly due to the low porosity of the granodiorite. However, changes in water content could be observed qualitatively.

In order to improve the detectability of the advancing moisture front, injection of $\mathrm{NaCl}$ brine upstream of the unsaturated front as a trace electrolyte was proposed. The TDR 
instrument was adapted for simultaneous measurement of water content (dielectric constant) and salt concentration (bulk electric conductivity). A laboratory experiment with a granodiorite column was conducted to test the fully automated equipment [Malicki et al., 1992]. The experiment demonstrated that changes in granodiorite water content and water salinity are detectable. In addition, it was concluded that salt diffusion in the liquid phase is negligible compared to the convective transport of the brine.

Based on the information obtained in the laboratory, a field-scale experiment was initiated to further study the transport of water and trace electrolytes through an unsaturated granodiorite matrix. The proposed test sequence includes periods of ventilation, brine injection, and water injection. The experiment is planned to conclude with a period of no ventilation to allow resaturation of the rock matrix.

In this report, results of scoping calculations of the planned ventilation and brine injection experiment are presented. The objective of this modeling study is to help design the main elements of the proposed test sequence, and to provide first estimates of the saturation and salt concentration to be expected at the observation points. The base-case parameter set is derived from measured data of the 1991/92 desaturation experiment using inverse modeling techniques [Finsterle, 1993b]. The uncertainty of the predicted parameters is evaluated by means of a linear error analysis. The inverse and forward modeling are both based on TOUGH2, ensuring the derived parameter set is consistent with the given model structure. 


\section{Experimental Layout}

The Unsaturated Zone Experiment (UZ) will be performed in the frontal chamber of the Ventilation Drift at the Grimsel Test Site (GTS), Switzerland. The geometrical layout of the drift and the boreholes as well as the major structural features is depicted in Figure 1 (after Vomvoris \& Frieg [1992]). Borehole BOVE 84.011, which is parallel to the drift at a distance of approximately $1.3 \mathrm{~m}$, has been selected for brine and water injection. Water potential, liquid saturation, and salt concentration are observed at four locations along the drift (St1 - St4), each equipped with six psychrometers and two tensiometers at different depths and up to six TDR-sensors for measuring the dielectric constant and the bulk electric conductivity.

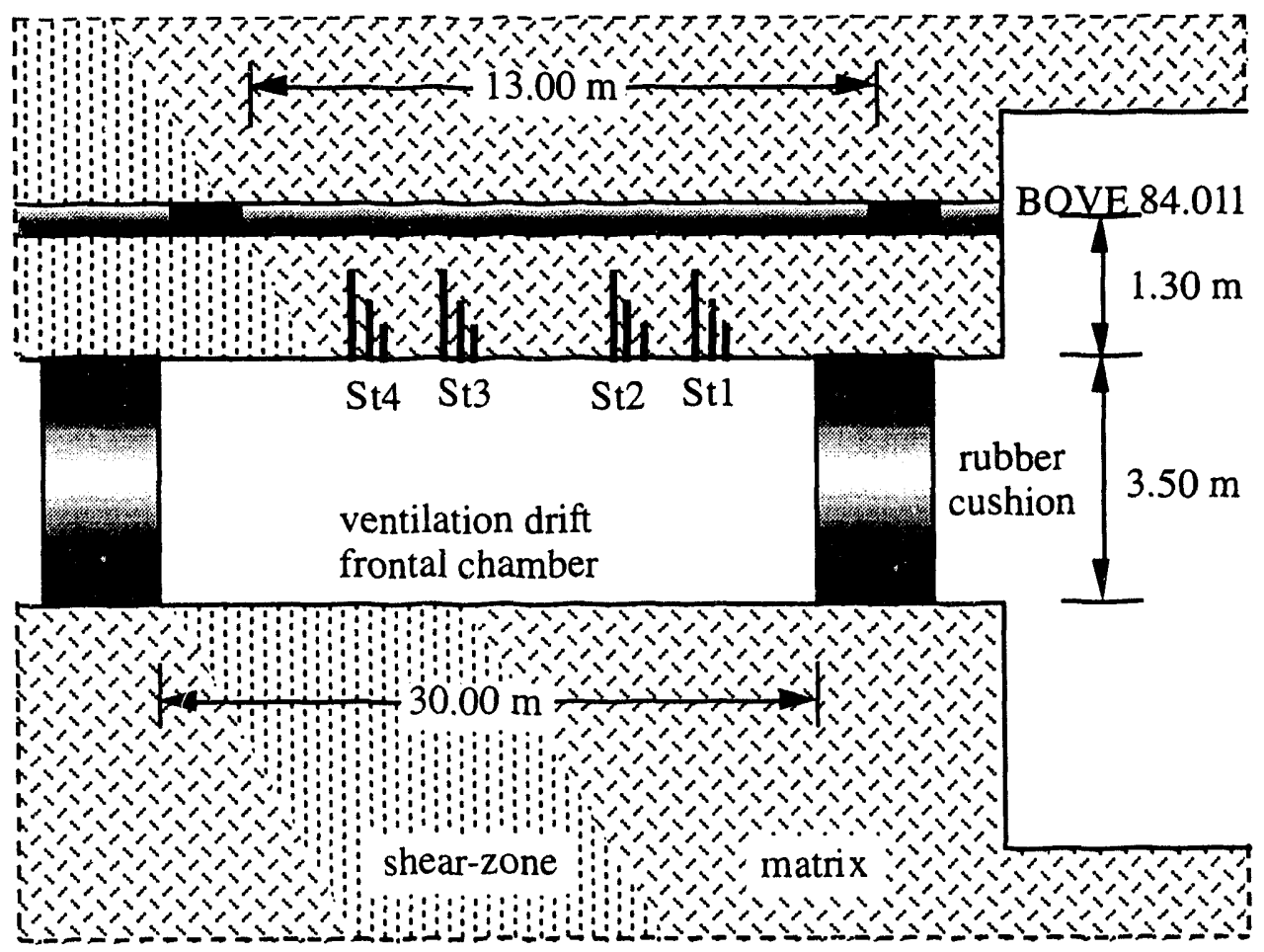

Figure 1: Schematic plan view of ventilation drift, borehole, and test equipment (not to scale) 
In accordance with the proposed test sequence, five periods are considered in the modeling study. Initially, the formation is believed to be fully liquid saturated. Starting January 1993, the relative humidity in the drift will be reduced by air-conditioning to less than $70 \%$. Desaturation of the rock matrix will occur for the next six months (Step 1). Subsequently, a 0.05 molar $\mathrm{NaCl}$ brine will be injected in borehole BOVE 84.011 at a constant rate of $5 \cdot 10^{-6} \mathrm{~kg} / \mathrm{s}$ for three days (Step 2), followed by a three-day perioc of fresh water injection at the same rate (Step 3). Two alternative procedures are being considered for the next step. In the first alternative (test sequence A), injection of water will be terminated while ventilation continues for another three months in order to observe the traced water transport toward the drift wall under ventilated conditions (Step 4A). This period would be followed by a resaturation experiment where the air in the drift is moisturized and kept at nearly $100 \%$ relative humidity (Step $5 \mathrm{~A}$ ). In the second alternative (test sequence $\mathrm{B}$ ), both injection and ventilation will be stopped after Step 3, and the propagation of the gas-brine and brine-water front would be observed during resaturation of the formation (Step 4B). The test sequence is summarized in Table 1.

\begin{tabular}{|c|c|c|c||}
\hline Step & Duration & Action in ventilation drift & Action in borehole BOVE 84.011 \\
\hline \hline 1 & 6 months & $\begin{array}{c}\text { ventilation } \\
\text { relative humidity }=68.5 \%\end{array}$ & $\begin{array}{c}\text { filled with fresh water } \\
\text { shut-in }\end{array}$ \\
\hline 2 & 3 days & $\begin{array}{c}\text { ventilation } \\
\text { relative humidity }=68.5 \%\end{array}$ & $\begin{array}{c}\text { brine injection } \\
\text { constant rate }=5 \cdot 10^{-6} \mathrm{~kg} / \mathrm{s}\end{array}$ \\
\hline 3 & 3 days & $\begin{array}{c}\text { ventilation } \\
\text { fresh water injection } \\
\text { constant rate }=5 \cdot 10^{-6} \mathrm{~kg} / \mathrm{s}\end{array}$ \\
\hline 4A & 3 months & $\begin{array}{r}\text { ventilation } \\
\text { relative humidity }=68.5 \%\end{array}$ & $\begin{array}{c}\text { filled with fresh water } \\
\text { shut-in }\end{array}$ \\
\hline 4B & 3 months & $\begin{array}{r}\text { no ventilation } \\
\text { relative humidity }=100 \%\end{array}$ & $\begin{array}{c}\text { filled with fresh water } \\
\text { shut-in }\end{array}$ \\
\hline
\end{tabular}

Table 1: $\quad$ Proposed test sequence 


\section{Modeling Approach}

\subsection{Fluid Properties and Physical Processes}

The proposed experiment leads to a flow system with a non-condensable gas and an aqueous phase of variable salinity. We have carried out model calculations using the TOUGH2 code [Pruess, 1991a] with equation-of-state module EOS7 for two-phase flow of saline water and air [Pruess, 1991b]. The saline mixture is described as consisting of a mass fraction $X_{b}$ of a reference brine, and a mass fraction $X_{w}=\left(1-X_{b}\right)$ of water. As the reference brine, a 0.05 molar $\mathrm{NaCl}$ solution is taken with a density $\rho_{b}=1004 \mathrm{~kg} / \mathrm{m}^{3}$ at $P_{0}=1$ bar and $\mathrm{T}_{0}=13^{\circ} \mathrm{C}$. To calculate liquid density, the mixing rule

$$
\frac{1}{\rho_{m}}=\frac{1-X_{b}}{\rho_{w}}+\frac{X_{b}}{\rho_{b}}
$$

is applied where $\rho_{w}$ is pure water density, $\rho_{b}$ is the density of the reference brine, and $\rho_{m}$ is mixture density. The relative change of density as a function of pressure and temperature for brine is assumed identical to the one of pure water:

$$
\frac{\rho_{b}(P, T)}{\rho_{b}\left(P_{0}, T_{0}\right)}=\frac{\rho_{w}(P, T)}{\rho_{w}\left(P_{0}, T_{0}\right)}
$$

Salinity effects on aqieous phase viscosity are modeled with a polynomial correction to the viscosity of pure water:

$$
\mu_{m}\left(P, T, X_{b}\right)=\mu_{w}(P, T) \cdot\left(1+a_{1} \cdot X_{b}+a_{2} \cdot X_{b^{2}}+a_{3} \cdot X_{b^{3}}\right)
$$

with $\mathrm{a}_{1}=4.82 \cdot 10^{-3}, \mathrm{a}_{2}=-2.77 \cdot 10^{-5}$ and $\mathrm{a}_{3}=7.81 \cdot 10^{-7}$, adapted from Pruess $[1991 \mathrm{~b}]$

Finally, dissolution of air in liquid is represented by Henry's law. Since air is less soluble in saline solutions than in pure water, Henry's constant linearly increases with increasing mixture molarity (for details see [Pruess, 1991b]). 
With the thermophysical properties defined above, the non-isothermal two-phase flow of gas and saline water is calculated in TOUGH2 by the integral finite difference method. While flow regions in single-phase liquid and two-phase conditions can be described, salt precipitation from supersaturated solutions is not taken into account. This effect may play an important role near the surface of the drift wall, where vaporization of the brine may cause a solid salt phase to evolve, possibly leading to a decrease of porosity and permeability. Furthermore, dispersion and diffusion in the liquid phase is not explicitly considered. However, there will be a substantial smearing of the gas-brine and brine-water interfaces due to numerical dispersion and capillary forces. Mechanical dispersion may be considered in future modeling studies using a dispersion module recently developed for the TOUGH2 simulator [Oldenburg \& Pruess, 1993].

The strong capillary pressures expected to arise from drying near the drift wall may lead to substantial vapor pressure lowering effects. As a consequence, water may not only be transported by viscous flow of liquid and humid air, but also due to vapor diffusion. However, vapor pressure lowering in the rock matrix which leads to concentration gradients as the driving force of binary diffusion, is neglected in this preliminary modeling study. Accordingly, diffusive transport of water and air in the gas phase is negligibly small. Water outflow from the rock occurs from capillary suction at the drift wall; drift air flows into the rock due to pressure drive. Finally, a potential Klinkenberg effect may lead to increased gas permeability in a tight rock matrix of low porosity. However, this effect is neglected for these design calculations. 


\subsection{Moisture Transfer at the Drift Surface}

Evaporation caused by reduced air humidity in the ventilated drift and the associated strong capillary suction pressures developing at the drift wall are the main processes driving the desaturation of the formation. The transfer of moisture at the drift wall is determined by the inflow of water from the porous rock, and by the diffusive flux of vapor into the drift.

Figure 2 provides a schematic view of the basic processes in the boundary layer near the rock surface. Water supplied from the formation in both liquid and gaseous phase is eventually transported across the rock surface toward the center of the drift by vapor diffusion, driven by the concentration gradient of vapor and mediated by an appropriate diffusion coefficient. If a laminar viscous boundary layer is formed, molecular exchange is the prevalent transfer mechanism. The thickness of the boundary layer is estimated to be on the order of centimeters [Watanabe, 1991] and depends on the wind velocity in the tunnel and the surface roughness of the drift wall. Further away from the wall, turbulent flow of air induced by wind shear stress greatly enhances the effective diffusivity of vapor.

The magnitude of the diffusion coefficient in the partially saturated porous medium is subject to speculation. While diffusive flow of vapor may be described as molecular diffusion restricted to the gas-filled fraction of the pore space $\left(\beta^{v}=\phi \cdot \tau \cdot S_{\mathrm{g}}\right)$, there is a great deal of evidence from studies in the soil science for enhanced vapor diffusion from pore-level phase change processes [e.g. Philip and de Vries, 1957; Cary and Taylor, 1962a,b; Hanks et al., 1967; Weeks et al., 1968; Jury and Letey, 1979; Ross, 1984]. In this study, we employ a pore geometry factor $\beta^{v}=0.5$ for enahnced vapor diffusion.

The different vapor diffusion coefficients described above lead to a relative humidity profile as shown in Figure 2. In the turbulent region of the drift, the relative humidity is more or less constant due to intense mixing. An almost linear increase of air humidity is assumed 
in the boundary layer. The relative humidity at the drift wall varies with the thickness of the boundary layer, but also with the effective permeability of the formation which is in turn a function of saturation and the characteristic curves of the formation. Local heterogeneities lead to variations in the evaporation rate and therefore partial drying of the drift wall.

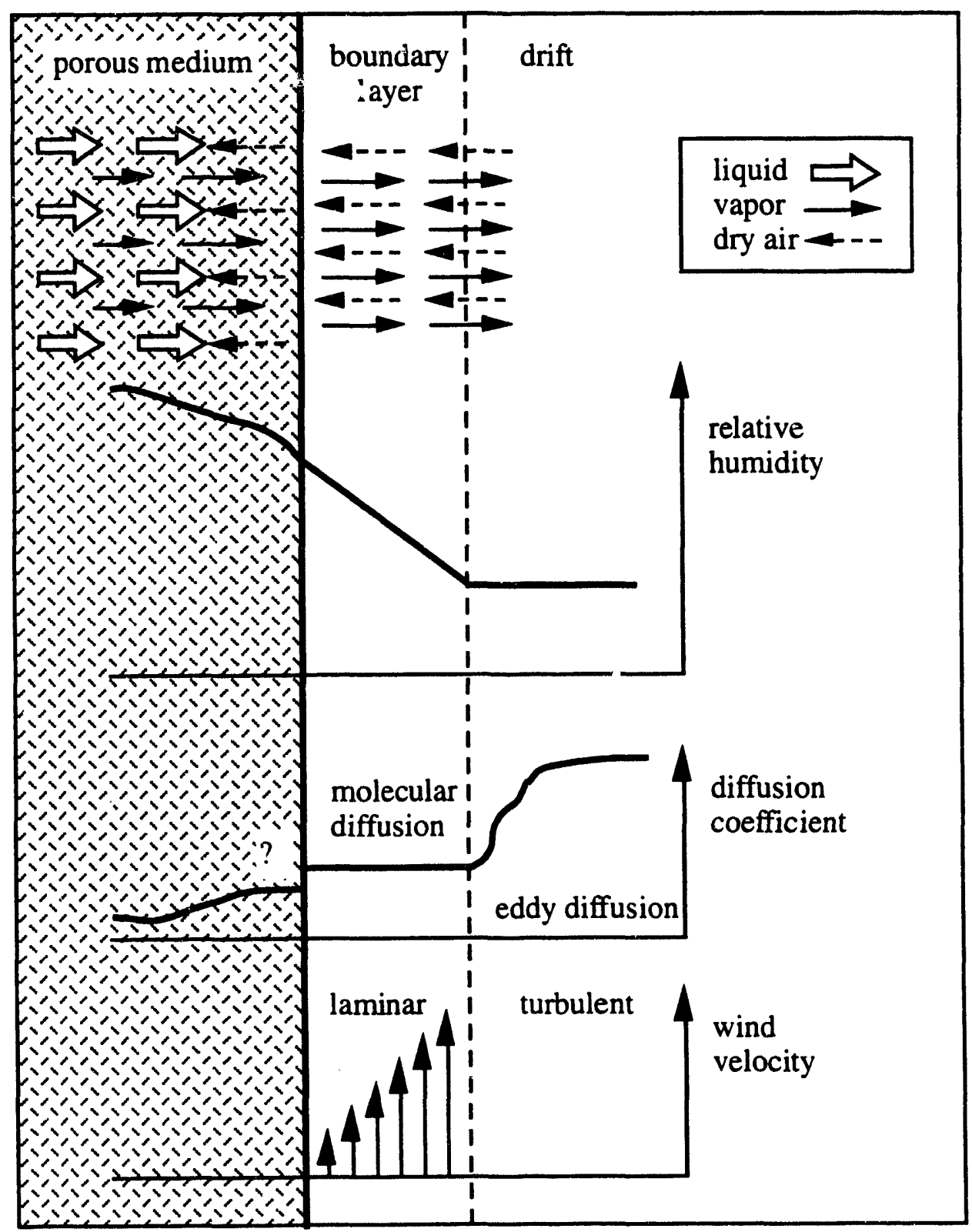

Figure 2: Schematic of moisture transfer at the drift wall 
In order to accurately predict the evaporation rate, it is important to know where exactly the drift climate is measured. If a mean value from the turbulent zone is provided, the diffusive moisture transfer across the boundary layer has to be considered to obtain the correct evaporation rate on the drift surface. Alternatively, it is suggested to measure relative air humidity at two points within the boundary layer in order to obtain a gradient of vapor concentration rather than a single value for relative humidity. Similar measurements were successfully performed in the GTS ventilation drift [Vomvoris \& Frieg, 1991] and in the validation drift at Stripa [Watanabe, 1991]. These measurements show increased evaporation rates near fractures.

A detailed model for bare surface evaporation is described in Connell \& Bell [1993]. In their work, the evaporation rate is calculated as a function of relative humidity, temperature gradient, wind velocity, surface roughness, and the Richardson number. In our numerical model, however, a more simplified approach is used wherein the relative humidity is assumed constant at the drift wall. The reduced humidity at the wall gives rise to an equivalent capillary suction pressure according to Kelvin's equation [Edlefsen \& Anderson, 1943]:

$$
\mathrm{P}_{\text {cap }}=\ln (\mathrm{h}) \cdot \rho_{\mathrm{l}} \frac{\mathrm{R} \cdot \mathrm{T}}{\mathrm{M}_{\mathrm{w}}}
$$

where $h$ is relative humidity, $\rho_{l}$ is the density of water, $T$ is absolute temperature, $R$ is the gas constant, and $\mathrm{M}_{\mathrm{w}}$ is the molecular weight of water. Modest reductions in relative humidity correspond to very strong capillary suction pressures. For example, for $T=286.15$ $K$ and a rele $y$ e humidity of $h=68.5 \%$, (4) yields $P_{\text {cap }}=-500$ bars. By applying a capillary suction pressure at the drift surface, the water potential in the drift is modeled which is strongly negative due to the reduced relative humidity. 


\subsection{Model Domail: and Computational Grid}

The computational grid for simulating the combined ventilation and brine injection experiment has to account for large saturation gradients near the drift as well as for large pressure, saturation, and brine concentration gradients near the injection borehole. In addition, the TP and TDR sensors have to be placed in proper location. For the preliminary scoping calculations presented here, a very simplified representation of the geometrical features was considered sufficient. Figure 3 shows the computational grid. Neglecting gravitational effects, a symmetry plane was assumed which intersects the center of the drift and the borehole. A higher grid resolution is applied near the drift wall and in the vicinity of the borehole. While the radial geometry of the drift was not reproduced, the location of the sensors was chosen such that the distances to both the drift and the borehole are equivalent to the actual distances. As a consequence, the configuration of the sensors in the coordinate system of the numerical model is somewhat different from the actual configuration.

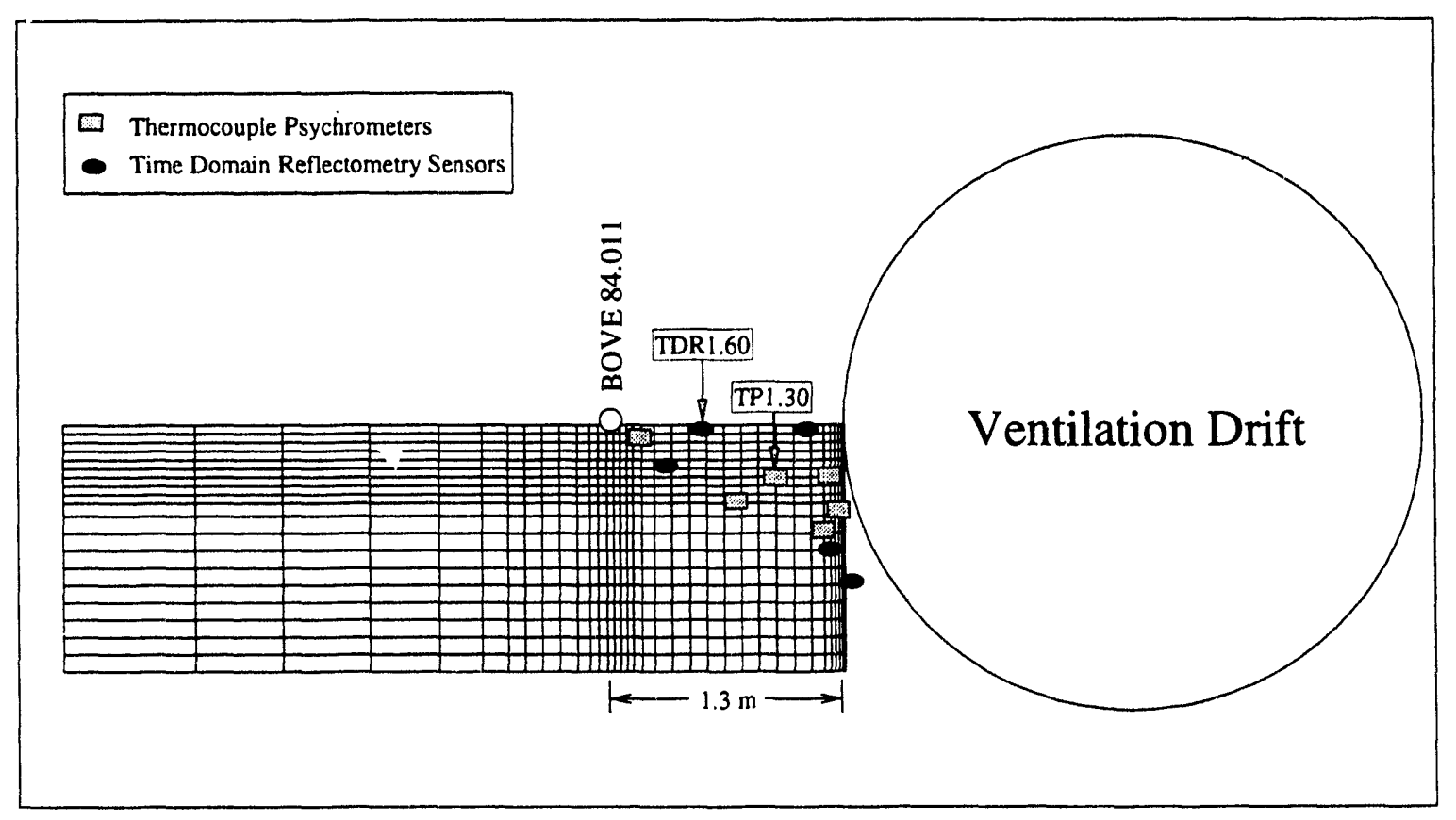

Figure 3: Model domain and computational grid 


\subsection{Formation Parameters, Initial and Boundary Conditions}

In the past, hydrogeologic formation parameters of the granodiorite at GTS were obtained from direct measurements at core samples, from the interpretations of several constant head and constant flow tests, and from modeling studies [Vomvoris \& Frieg, 1992]. For this study, model parameters have been calibrated by inverse modeling methods [Finsterle, 1993] from the data collected during the 1991 desaturation experiment [Gimmi et al., 1992]. The most important parameters are the absolute permeability of the matrix, and two parameters of van Genuchten's relative permeability and capillary pressure function, namely $n$, which corresponds to the pore size distribution index, and $\alpha$, which can be related to the air entry pressure.

To implement boundary conditions at the drift wall, an additional column of grid blocks with a very small nodal distance of $\mathrm{d}_{01}=10^{-10} \mathrm{~m}$ was added to the right of the grid shown in Fig. 3. These grid blocks were made "inactive", so that their thermodynamic conditions would remain unchanged during a simulation. The conditions in these grid blocks were chosen to represent the assumed drift-wall conditions of $P_{\text {gas }}=1$ bar, $P_{\text {cap }}=-500$ bar (corresponding to a relative humidity of $h=68.5 \%$ ), $\mathrm{k}_{\mathrm{r}, \mathrm{gas}}=1$, and $\mathrm{k}_{\mathrm{r}, \mathrm{liq}}=0$. Accordingly, resaturation periods are modeled by applying $P_{c a p}=0$ at the drift-wall boundary, assuming that the air in the drift is kept vapor saturated by means of moisturizers. The conditions in the drift-wall boundary blocks were achieved by assigning the blocks to a special flow domain with appropriate relative permeability and capillary pressure functions which provide the desired relative permeability and capillary suction for a fixed gas saturation.

Note that in our simulations vapor pressure lowering effects are represented only indirectly, by assigning a capillary suction pressure that is equivalent to a humidity of $68.5 \%$ to the drift wall. Moisture transfer from the rock to the drift occurs as liquid phase flow, driven by this strong capillary suction. Vapor pressure is equal to saturated vapor pressure 
everywhere, so that vapor mass fraction in the gas phase only varies in response to small transient changes in total gas phase pressure. Therefore, moisture transfer from vapor diffusion is negligibly small. In future work we plan to fully account for vapor pressure lowering effects, and to examine simultaneous moisture transfer in both liquid and gaseous phases driven by consistent capillary and humidity conditions throughout.

Under fully liquid saturated conditions in the formation, a pressure of 0.7 bar above atmospheric was observed in borehole BOVE 84.011 [Vomvoris \& Frieg, 1992]. In the model, we maintain a constant pressure of 3.7 bar at a distance of $5.0 \mathrm{~m}$ to achieve a steadystate profile matching the observed borehole pressure. The initial pressure and saturation distribution prior to ventilation was obtained by running the model to steady state. Dissolved gas comes out of solution and formes a free gas phase with a gas saturation of less than 0.002 near the drift. While the actual conditions are unknown, assigning a low initial gas saturation is justified by the fact that a residual gas content is likely to be in place due to previous desaturation experiments periormed at the same site.

Measurements of negative water potentials at a depth of $2,5,10,20,40$, and $80 \mathrm{~cm}$ monitored during a period of three months were used to calibrate the model. The calibration was performed by inverse modeling using the ITOUGH2 code [Finsterle, 1993]. The best fit achieved is shown in Figure 4. The corresponding characteristic curves are depicted in Figure 5. No effort has been made to match the data perfectly. However, the parameter set obtained from inverse modeling of the 1991 ventilation test is believed to be reasonable also for the planned desaturation experiment because of the similarity in both the scale of the problem and the main physical processes. In addition, the parameters can be considered consitent and optimal for the given model structure, i.e. model geometry, discretization, and boundary conditions. A better fit of the same data can be obtained using radial geometry and a time dependent vapor pressure boundary condition to account for changes in the drift climate. 


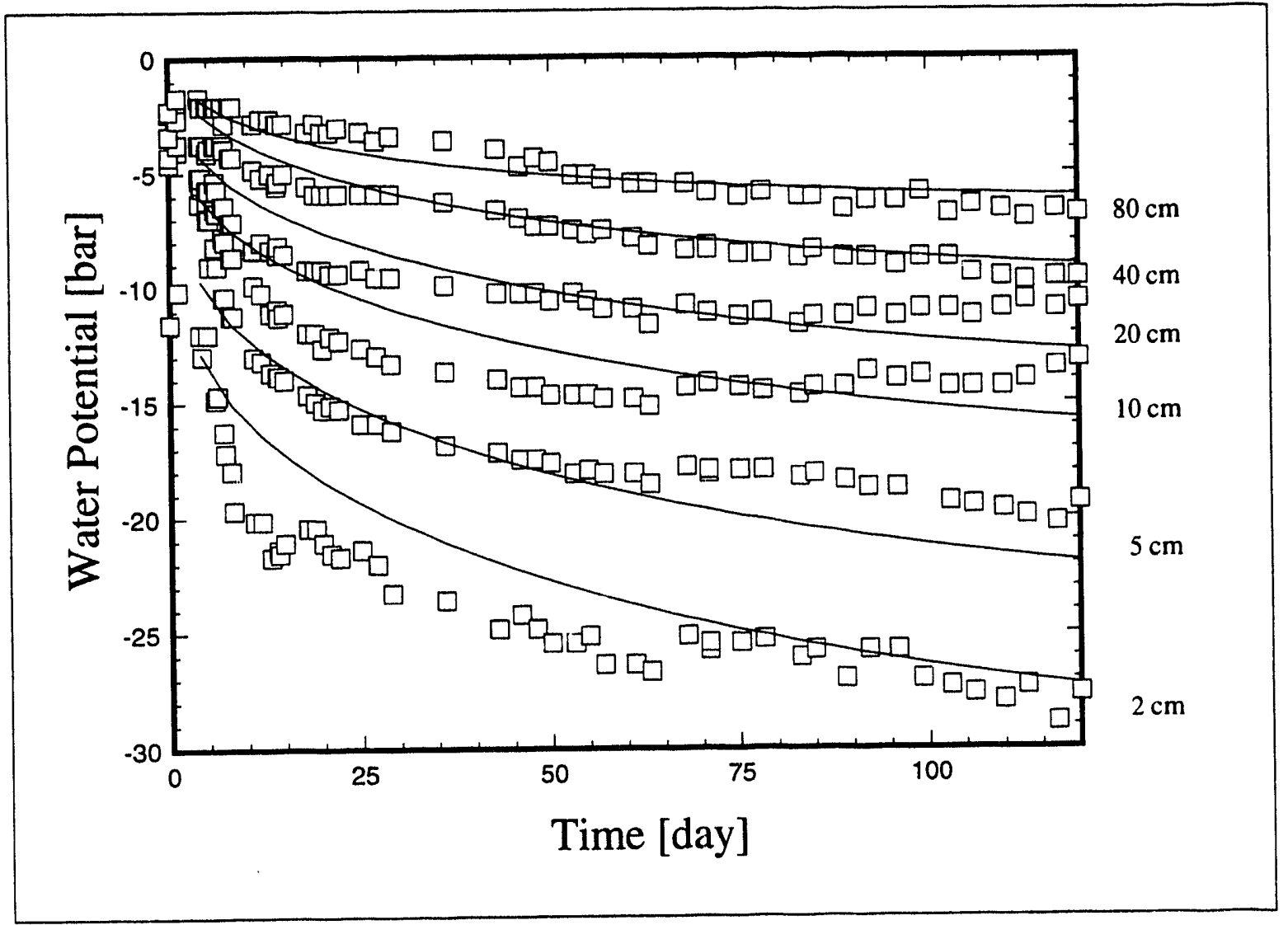

Figure 4: Comparison between measured (symbols) and calculated (solid lines) water potentials during desaturation experiment
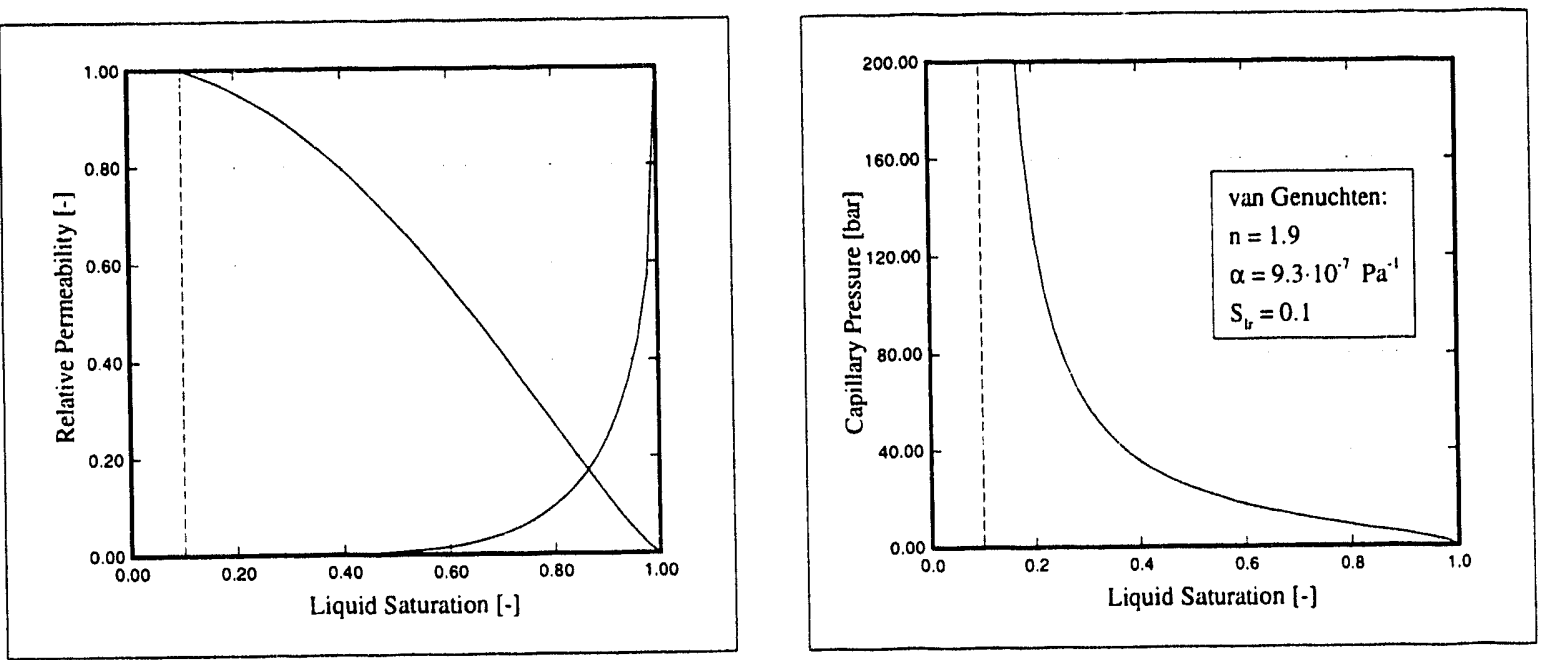

Figure 5: Characteristic curves determined from inverse modeling of desaturation experiment 
Table 2 summarizes model parameters, including data used for a modified model with a high permeability fracture zone (see below).

The absolute permeability of $6.8 \cdot 10^{-19} \mathrm{~m}^{2}$ determined by inverse modeling is about 2.5 times lower than the value obtained from water balance considerations, but is within the range of the estimates from welltest interpretations and trial and error modeling efforts [Vomvoris \& Frieg, 1992]. The water balance measurements were obtained from covering the shear zone with plastic sheeting and collecting the water at the base of the wall. Flux from the matrix is calculated as the difference between the total inflow and the water collected from the shear zone. The hydraulic conductivity is estimated using Thiem's formula [Thiem, 1906]. This method provides an average conductivity of the granodiorite section of the ventilation drift and may thus be different from the estimate obtained on a local scale. The parameters $\mathrm{n}=1.9$ and $\alpha=10^{-6} \mathrm{~Pa}^{-1}$ of van Genuchten's model are in reasonable agreement with the values obtained by fitting a bi-modal water retention curve to laboratory data obtained from granodiorite core samples [Durner, personal communication].

In order to study the impact of possible preferential flow paths on the brine and saturation distribution, a permeable fracture zone was introduced in the model domain normal to the drift wall, bypassing the borehole at a distance of $0.2 \mathrm{~m}$. The thickness of the fracture zone is $0.05 \mathrm{~m}$. Its permeability is assumed five times higher than matrix permeability. The air entry pressure of van Genuchten's capillary pressure function is $1 / \alpha_{f}=-5.0$ bar. This choice of absolute permeability and capillary pressure for the fracture zone is consistent with Leverett's model which states that the ratio of capillary pressures is inversely proportional to the square root of the permeability ratio. The zone with higher permeability and lower capillarity, referred to as "fracture zone", is conceived to model small scale heterogeneities; it should not be seen as a geologically identifiable structure like the VE shear-zone. 


\begin{tabular}{|c|c|c|}
\hline Parameter & Value & Unit \\
\hline matrix absolute permeability $\mathrm{k}_{\mathrm{abs}, \mathrm{m}}$ & $6.8 \cdot 10^{-19}$ & $\mathrm{~m}^{2}$ \\
\hline fracture zone absolute permeability $k_{a b s, f}$ & $3.4 \cdot 10^{-18}$ & $\mathrm{~m}^{2}$ \\
\hline fracture zone thickness & 0.05 & $\mathrm{~m}$ \\
\hline porosity $\phi$ & 0.01 & - \\
\hline tortuosity factor $\tau$ (for air diffusion) & 0.50 & - \\
\hline pore geometry factor $\beta$ (for enhanced vapor diffusion) & 0.50 & - \\
\hline \multicolumn{3}{|c|}{ Matrix: Parameters for van Genuchten's Characteristic Curves } \\
\hline residual liquid saturation $S_{1 r}$ & 0.10 & - \\
\hline residual gas saturation $\mathrm{S}_{\mathrm{gr}}$ & 0.00 & - \\
\hline pore size distribution $n$ & 1.88 & - \\
\hline matrix air entry pressure $(1 / \alpha)_{\mathrm{m}}$ & $1.0 \cdot 10^{6}$ & $\mathrm{~Pa}$ \\
\hline fracture zone air entry pressure $(1 / \alpha)_{\mathrm{f}}$ & $5.0 \cdot 10^{5}$ & $\mathrm{~Pa}$ \\
\hline \multicolumn{3}{|c|}{ Boundary Conditions } \\
\hline relative humidity in drift $h$ & 0.685 & - \\
\hline corresponding equivalent liquid suction pressure & $-5.0 \cdot 10^{7}$ & $\mathrm{~Pa}$ \\
\hline constant pressure at drift wall & $1.0 \cdot 10^{5}$ & $\mathrm{~Pa}$ \\
\hline constant pressure $5 \mathrm{~m}$ from drift wall & $3.7 \cdot 10^{5}$ & $\mathrm{~Pa}$ \\
\hline \multicolumn{3}{|l|}{ Initial Conditions } \\
\hline steady-state pressure profile between boundaries & linear & $\mathrm{Pa}$ \\
\hline temperature $T$ & 13 & ${ }^{\circ} \mathrm{C}$ \\
\hline initial gas saturation $\mathrm{S}_{\mathrm{g} 0}$ & $\sim 0.001$ & - \\
\hline
\end{tabular}

Table 2: Formation parameters, boundary and initial conditions 
Fluid flow in the homogeneous medium is discussed in Section 3.5. Results of the heterogeneous case with a discrete fracture zone are presented in Section 3.6

A covariance matrix for five of the estimated input parameters has been calculated based on the data from the 1991 desaturation experiment. The five parameters are the logarithm of the absolute permeability, the residual liquid saturation and the two parameters $n$ and $\log (1 / \alpha)$ of van Genuchten's characteristic curves, and the relative humidity in the drift, which is considered uncertain due to the simplifications made in the numerical model (see Section 3.2).

The variances and correlation coefficients in Table 3 show that the water potentials are not very sensitive to the residual liquid saturation $S_{l r}$ and the relative humidity $h$. As a result, these two parameters cannot be accurately determined from the available data. On the other hand, only small variances are obtained for the two parameters $\log (1 / \alpha)$ and $n$. However, the latter two are highly correlated. It should be emphazisized that these correlations originate from the data being used for calibration but are also inherent to van Genuchten's model. The covariance matrix is later used to predict the uncertainty of the model results by means of a simple First-Order-Second-Moment error analysis defined by

$$
\mathbf{C}_{\mathrm{x}}=\mathbf{J} \cdot \mathbf{C}_{\mathrm{p}} \cdot \mathbf{J}^{\mathrm{T}}
$$

where $\mathbf{C}_{\mathbf{x}}$ is the covariance matrix of the model predictions at certain points in space and time, $\mathbf{C}_{\mathrm{p}}$ is the covariance matrix of the input parameters (see Table 3 ), and $\mathbf{J}$ is the Jacobian matrix with elements $\mathrm{J}_{\mathrm{ij}}=\partial \mathrm{x}_{\mathrm{i}} / \partial \mathrm{p}_{\mathrm{j}}$. The variances of the matrices $\mathbf{C}_{\mathrm{x}}$ and $\mathbf{C}_{\mathrm{p}}$ lower bounds of the actual values because nonlinearity effects and the uncertainty of other correlated parameters are neglected. Finally, and most importantly, it is assumed that the model is capable of exactly reproducing the real system behavior; no systematic errors from incomplete process description or inadequate model structure are taken into account when 
calculating confidence regions. These assumptions are being tested by the experiment. The brine travel time, for example, will be measured in the field and can thus be used to support or negate the model assumptions, including the parameter values obtained from inverse modeling.

\begin{tabular}{||c||c|c|c|c|c||}
\hline & $\log \left(\mathrm{k}_{\mathrm{abs}}\right)$ & $\mathrm{S}_{\mathrm{lr}}$ & $\mathrm{n}$ & $\log (1 / \alpha)$ & $\mathrm{h}$ \\
\hline \hline $\log \left(\mathrm{k}_{\mathrm{abs}}\right)$ & 0.062 & 0.467 & -0.752 & -0.865 & -0.032 \\
\hline $\mathrm{S}_{\mathrm{lr}}$ & 0.036 & 0.098 & -0.812 & -0.842 & 0.018 \\
\hline $\mathrm{n}$ & -0.024 & -0.033 & 0.017 & 0.924 & 0.150 \\
\hline $\log (1 / \alpha)$ & -0.019 & -0.023 & 0.010 & 0.008 & 0.014 \\
\hline $\mathrm{h}$ & -0.002 & 0.001 & 0.002 & 0.002 & 0.056 \\
\hline
\end{tabular}

Table 3: Variances (diagonal), covariances (lower triangle), and correlation coefficients (upper triangle) of TOUGH2 input parameters

\subsection{Simulation Results for Homogeneous Medium}

Initially, the formation is essentially fully liquid saturated with a steady water inflow to the drift of $3 \cdot 10^{-7} \mathrm{~kg} / \mathrm{s}$ per meter. With ventilation, the humidity at the drift-wall surface is reduced to $68.5 \%$, leading to an equivalent liquid suction pressure of about -500 bar according to Kelvin's equation. As water is removed from the rock, gas enters and a rather smooth gas-liquid front propagates into the formation. It should be pointed out that no sharp interfaces can be identified. The zone of significantly increased gas saturation, e.g. $\mathrm{S}_{\mathrm{g}}>0.50$, penetrates only a few centimeters into the formation while a relatively large region is only moderately affected by the drying out (see Figures 6 and 7 below). The actual shape 
of the saturation profiles strongly depends on the characteristic curves which are not well known. The gas pressure throughout the partially saturated zone is close to atmospheric. The presence of a low pressure region has been observed in two parallel boreholes at GTS (BOVE 84.011 and BOVE 84.018), leading to speculation about the impact of the unsaturated zone on head and inflow measurements [Vomvoris \& Frieg, 1992]. Low pressures are caused by the removal of liquid phase by capillary suction.

After six months of ventilation, the simulation shows a total water inflow to the drift of $1.2 \cdot 10^{-6} \mathrm{~kg} / \mathrm{s}$, indicating that more water is removed than supplied by the formation under steady-state conditions. This implies that the unsaturated zone is still developing; no equilibrium has yet been reached. (For a more detailed discussion of inflow behavior to a ventilated drift see also [Finsterle \& Vomvoris, 1991].) The results of the test sequence are qualitatively depicted in Figure 6, where brine content and gas saturation are visualized at the end of each test step. Here, brine content is defined as the product of liquid saturation and brine mass fraction in the liquid phase. This quantity gives the fraction of brine present in the pore space. In all figures, the level of the leftmost contour is $S_{g}=0.05$; the increment between the contours is 0.05 . 

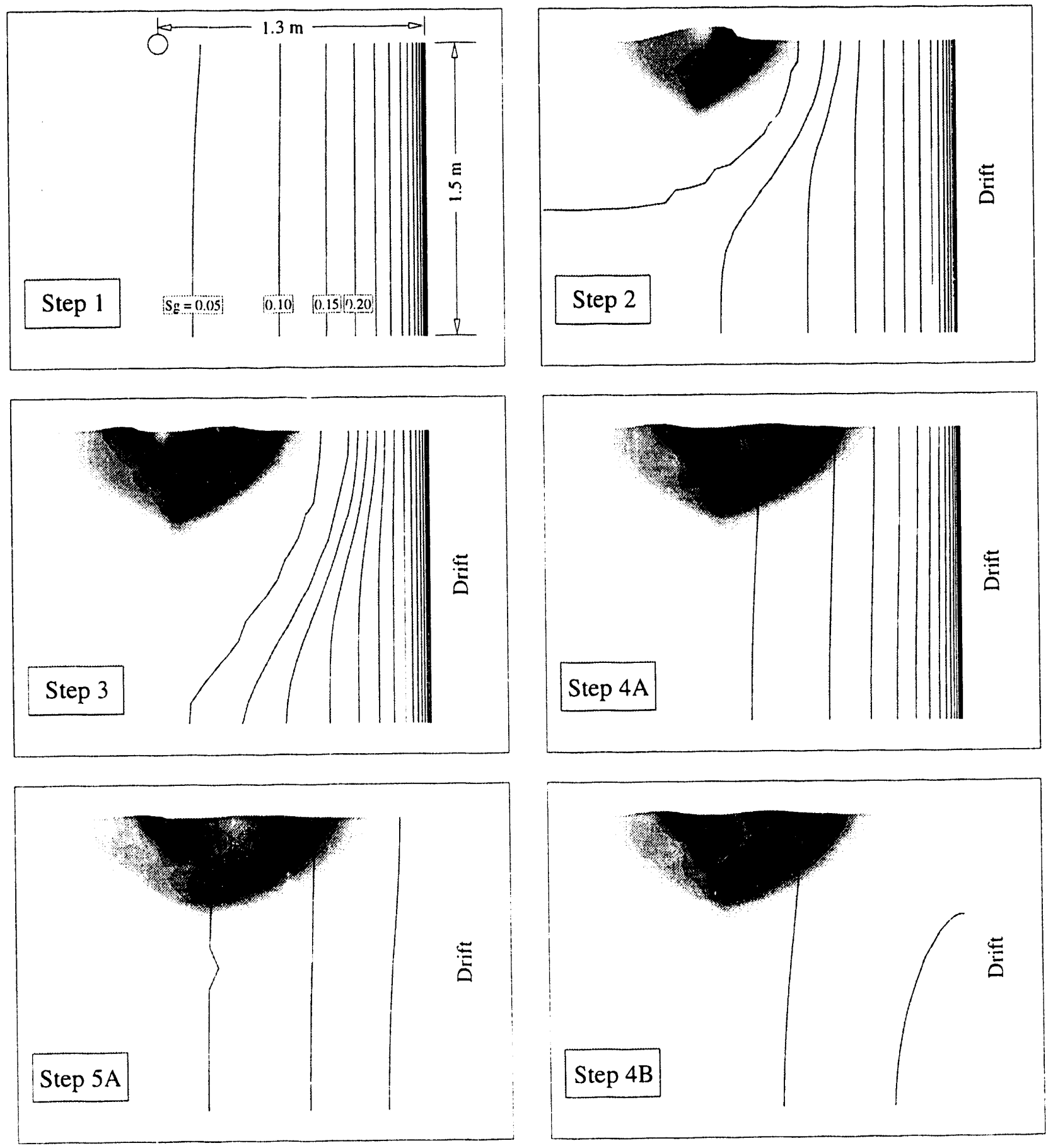

Figure 6: Brine content (shaded surface) and gas saturation (contours), homogeneous medium 
In Step 2, a 0.05 molar $\mathrm{NaCl}$ brine is injected at a constant rate of $5 \cdot 10^{-6} \mathrm{~kg} / \mathrm{s}$ for a period of three days. The gas in the vicinity of the borehole is displaced and compressed because of the high injection pressure which reaches 47 bars at the end of Step 3. Lower injection rates may be more convenient to reduce the rather high injection pressure. In this case, the injection period should be appropriately prolonged in order to maintain the total amount of injected brine (1.3 $\mathrm{kg}$ per meter borehole). In reality, dispersion effects due to capillarity, heterogeneity in terms of permeability and suction pressure, and flow instabilities may lead to a smeared front between the injected brine and the displaced gas-water mixture; the smearing seen in the simulation results mainly stems from capillary pressure effects and numerical dispersion.

Injection of fresh water in Step 3 creates a composite system with three concentric zones. The region nearest to the injection point contains fresh water, followed by an intermediate ring zone of highly saline water which continues to displace the mixture of formation water and air. These regions are not circular but slightly distorted because of the background flow field and capillary forces which tend to suck liquid mainly in the direction of the drift.

After injection is shut-in, the unsaturated zone resumes developing (Step 4A). It is interesting to note that the brine content does not seem to be significantly affected by the propagation of the gas-liquid front even though large capillary pressure gradients are acting across the interface between the saturated and the unsaturated zone. This can be explained by strong phase interferences leading to very low liquid relative permeabilities even at modest gas saturations (see characteristic curves in Figure 5). Furthermore, the change of gas saturation is too modest to initiate a self-sharpening effect which could be due to evaporation and brine accumulation at the gas-liquid front in addition to increased flow resistance for the liquid phase. However, a comparison of the numerical results of Step 4A and Step 4B 
reveals the impact of the capillary forces on the brine transport. If ventilation continues after fluid injection (Step 4A), capillarity acts as an additional driving force, leading to a slightly faster transport of brine toward the drift. However, the difference is too modest to be seen in Figure 6.

During resaturation (Step 4B and 5A, respectively) it can be seen that the velocity of the saline water is lower than the velocity with which liquid saturation increases. This indicates that resaturation is not a purely hydromechanical process where the gas phase is displaced by inflowing formation water. Most of the drained pore volume is refilled due to redistribution of the liquid phase, driven by capillary forces. In comparison, the convective flow of formation water is minor because of the very low permeability. As a result, changes of water content may not be indicative of the velocity with which water flows toward the drift under two-phase or even saturated conditions. Figure 7 demonstrates the resaturation process showing gas saturation profiles at different times. The profiles are not simply shifted toward the drift with time, as one would expect from a piston displacement model. After three months, the effect of moisturizing the drift air can be seen only to a distance of about $0.5 \mathrm{~m}$ whereas gas that has penetrated deeper into the formation is only slowly reduced by dissolution and displacement. 


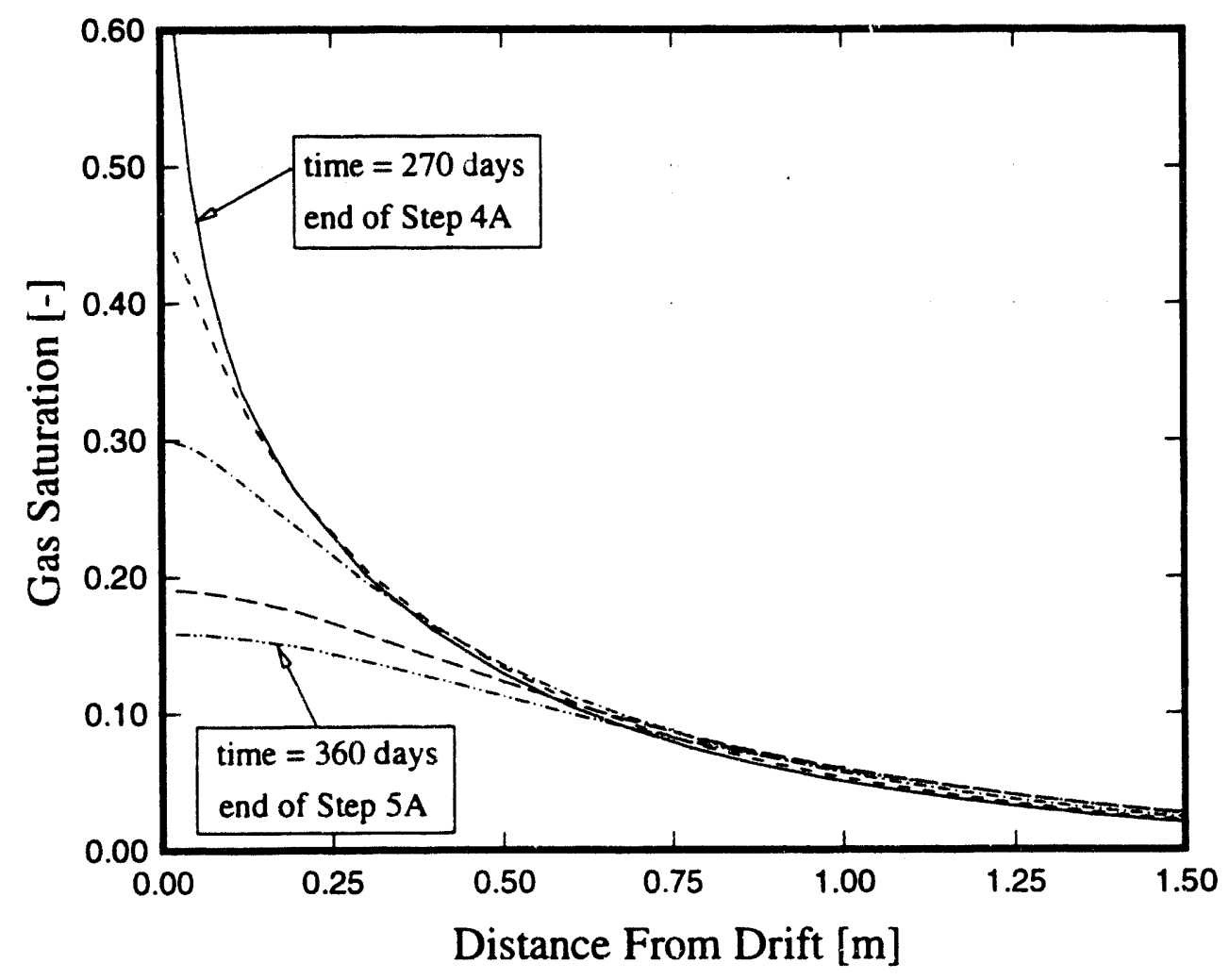

Figure 7: Gas saturation profiles during resaturation (Step 5A)

Predictions of the observable system response at two locations, assuming that test sequence $\mathrm{A}$ is carried out, are discussed in the remainder of this section. First, the brine content is calculated for the TDR 1.60 sensor which is located 0.6 meters from the drift wall and about 0.7 meters from the injection borehole. The results plotted in Figure 8 show the relative brine content as a function of time. 


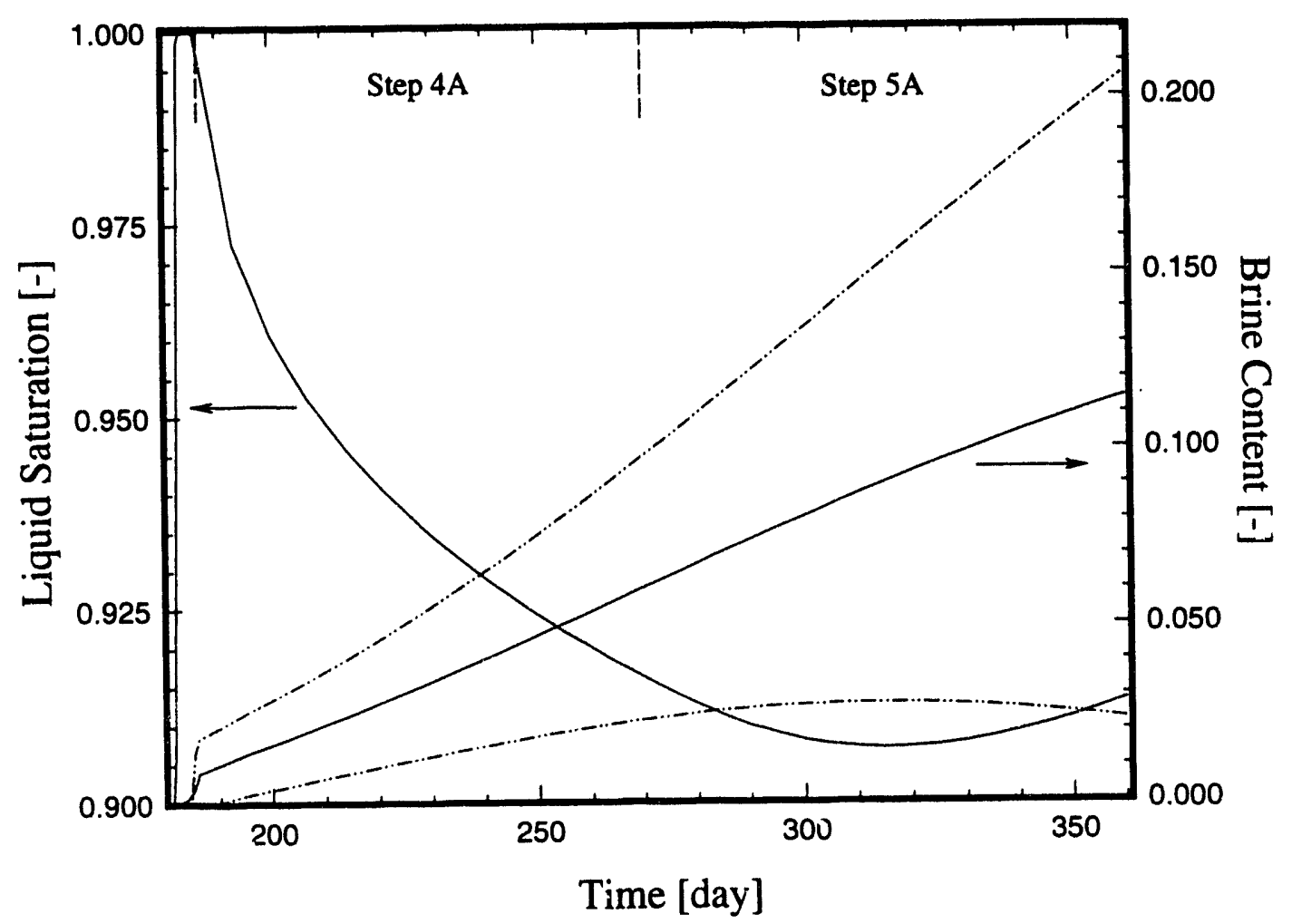

Figure 8: Relative brine content and liquid saturation at sensor TDR1.60: homogeneous medium. The dash-dotted lines indicate the $95 \%$ confidence band for predicted brine content.

During the initial ventilation period (Step 1, not shown), the liquid saturation observed at TDR1.60 decreases to a value of 0.9 . Subsequently, the pore space is refilled with liquid due to the injection of water and brine. The displaced gas phase invades again during the Step 4A ventilation period. An increase of liquid saturation as a result of the humid air conditions during test Step $5 \mathrm{~A}$ can be seen with a delay of about six weeks. Relative brine concentration increases almost linearly to about 0.12 . The slope of the curve is strongly influenced by the shape of the brine front which is controlled by the capillary pressure functions and numerical dispersion. It can be estimated that another 6 months of resaturation is needed to observe the maximum brine concentration passing the sensor, followed by the 
fresh water injected during Step 3. The magnitude of the maximum brine content which may be critical for the detectability of the brine pulse again depends on the dispersion processes mentioned above. An optimistic - still rather large - uncertainty range was calculated assuming linear error propagation, and is indicated by the $95 \%$-confidence error band.

Finally, the system response at sensor TP1.30 is depicted in Figure 9. The TP sensor is located $0.3 \mathrm{~m}$ from the drift wall and $1.05 \mathrm{~m}$ from borehole BOVE 84.011 . It monitors the negative water potential as a function of time.

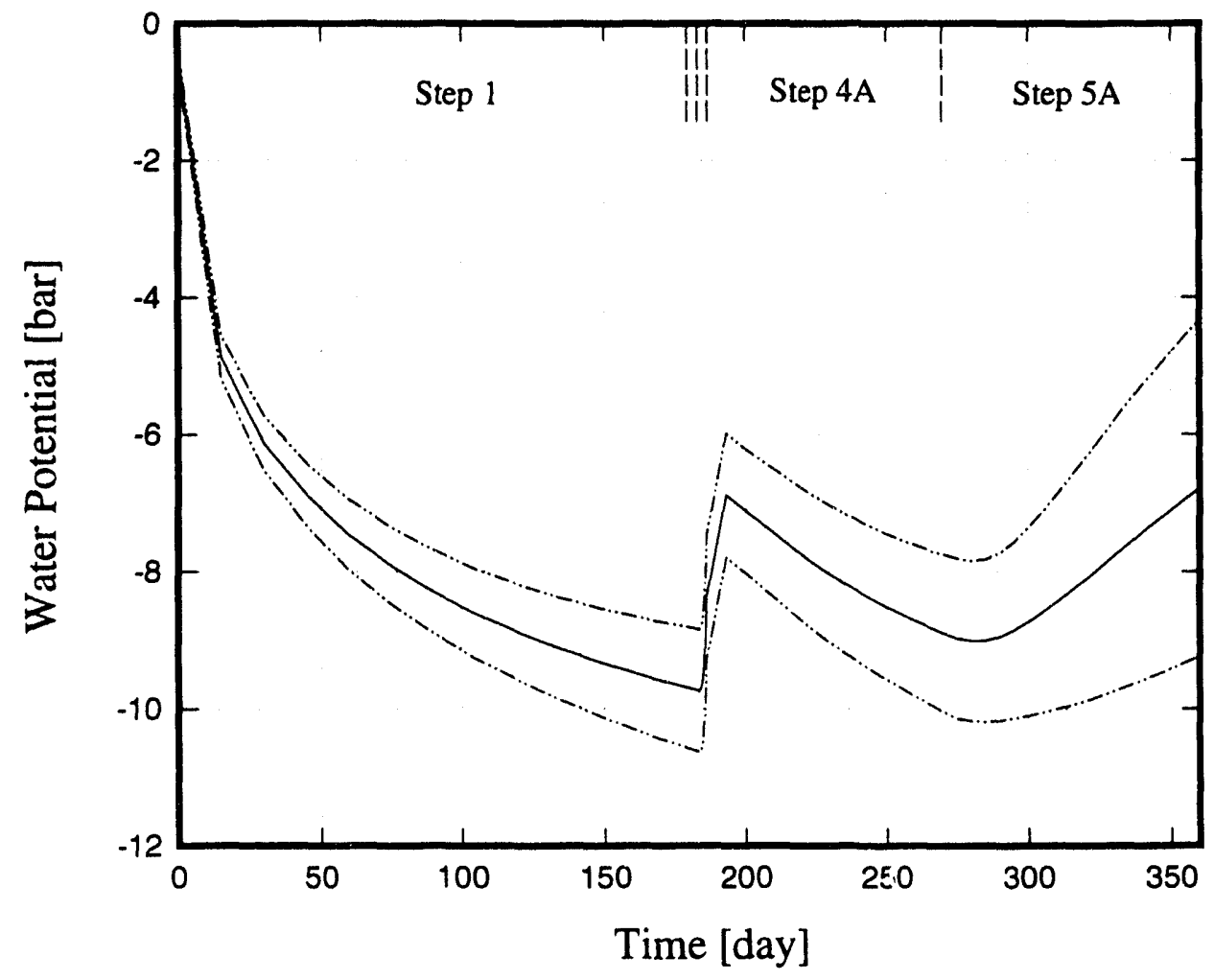

Figure 9: Water potential at sensor TP1.30, homogeneous medium

As mentioned in Section 3.4, the model has been calibrated using data from TP sensors during the 1991 desaturation experiment. Therefore, the predicted response of the TP1.30 sensor during Step 1 exhibits a similar behavior as the one shown in Figure 4. When 
brine and water is injected (Step 2 and Step 3, respectively), liquid saturation and water potential increase almost instantaneously. The slope of the liquid saturation and water potential curve during Step 4A and Step 5A indicate similar velocities for both, desaturation and resaturation. However, hysteresis may play a dominant role when reversing flow direction leading to a much slower resaturation of the void pore space than predicted in these simulations.

In this section, the behavior of a homogeneous system has been discussed on the basis of a simplified numerical model. It can be concluded that for the assumed model with its low permeability and weak natural pressure gradient, the propagation of the brine is mainly driven by the applied injection pressure and the perturbation of the suction pressure gradient in the vicinity of the borehole. As a result, the information obtained from the TDR sensors may not reflect the true velocity of the liquid phase in a two-phase environment near a ventilated drift. Furthermore, the moisture content in the formation strongly depends on the climatic conditions in the drift. A change of the negative water potential measured by Thermocouple Psychrometers may be caused by enhanced vapor content in the drift. Refill of void pore space by formation water or traced fluid is a much slower process. Consequently, a simultaneous increase of brine content and water potential is not likely to be observed even if test sequence $B$ is chosen. Finally, the absolute values of the predicted brine contents are highly dependent on the choice of the capillary pressure function. Numerical as well as mechanical dispersion effects may also be important, so the model results have to be interpreted with caution. They provide good insight into the physical processes and may indicate the overall pattern of brine distribution. However, no quantitatively accurate prediction should be expected from these simulations. 


\subsection{Simulation Results for Heterogeneous Medium}

Heterogeneity may induce preferential flow of brine, leading to a bypass or an early response of some of the sensors. Heterogeneity is modeled by introducing a linear feature, perpendicular to the drift, with higher permeability and lower air entry pressure compared to that of the surrounding rock matrix. The parameter values used to characterize this "fracture zone" are given in Table 2.

Provided that the effective permeability of the fracture zone is relatively low so that evaporation rate is higher than water supply from the formation, drying occurs preferentially along the fracture which is drained by the high capillary forces in the adjacent matrix blocks (see saturation distribution after six months of ventilation in Figure 10). In this case, the unsaturated zone propagates deeper into the formation if a low capillary pressure feature is present. This is in contradiction with the observations in the VE drift where high permeable zones remain wet during ventilation periods [Vomvoris \& Frieg, 1991]. However, it should be realized that the system behavior is governed by several interacting factors. These are basically the ratio of capillary pressures in the matrix and the fracture zone, and the effective permeability and ambient pressure gradient compared to the evaporation rate. Due to the complex interaction of the processes previously discussed in Section 3.3, it is difficult to predict the pattern of saturations and flow rates in a heterogeneous medium, if the characteristic curves and the vapor transfer mechanism are not well known. Higher pressure gradients, lower suction pressures in the matrix, and lower evaporation rates may in fact keep the fracture zone wet during ventilation. In the numerical model, a constant suction pressure of -500 bar is applied at the boundary to both the matrix and the fracture zone, simulating reduced vapor pressure at the drift wall during ventilation. This simplified approach does not account for the possibility of an increased relative humidity in those portions of the drift wall that are intersected by a fracture. 
Figure 10 qualitatively shows the impact of a highly permeable feature on brine content and saturation distribution. Using the concepts and parameters mentioned above, ventilation leads to higher gas saturations in the fracture. Brine and water injection occurs at a distance of $0.2 \mathrm{~m}$ from the highly permeable feature (Step 2 and 3), reducing gas saturation in both the fracture and the matrix. In Step 4, brine is swept along the highly conductive zone. However, if ventilation continues (Step 4A), this is counteracted by the reduction of the liquid saturation in the fracture, leading to small liquid relative permeabilities and reduced brine content. In fact, if ventilation is strong enough to keep the fracture at very low liquid saturations, it acts as a barrier for brine transport. Brine flowing along the fracture zone also diffuses into the matrix by means of capillary suction, reducing the velocity of the brine front. A slightly different picture results if ventilation stops (Step 4B). However, as already discussed in Section 3.5, increased relative humidity in the drift mainly leads to higher liquid saturations near the drift wall, whereas the saturation conditions in the vicinity of the borehole are nearly unchanged. The unsaturated zone penetrates along the fracture zone due to redistribution of liquid driven by capillary forces, even if ventilation ceases (compare Step 3 and Step 4B). As a result, the fracture zone at a certain depth remains unsaturated for a long period of time which reduces its importance as a preferential flow path for brine. On the other hand, if the effective liquid permeability is high enough to sweep most of the brine along the fracture zone, increased brine contents are expected downstream. In this case, a barrier effect can be observed for flow normal to the fracture. The strength of these counteracting factors again depends on the ratio of the capillary forces in the fracture and the matrix, as well as on the effective permeabilities and the evaporation rate. The parameter combination selected for this study leads to preferential flow along the fracture. However, the effect is somewhat weakened due to reduced effective permeability in the fracture zone. 

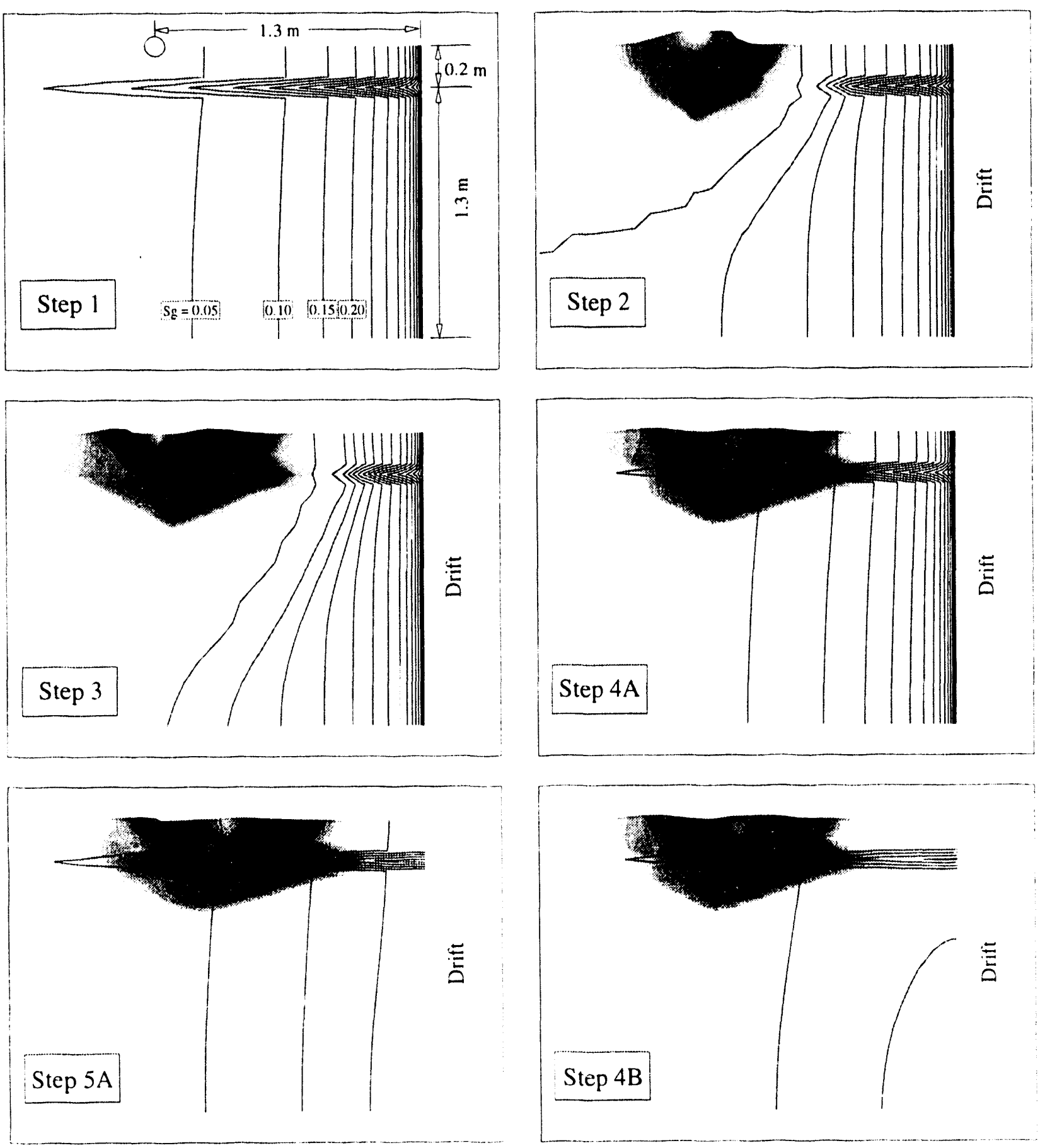

Figure 10: Brine content (shaded surface) and gas saturation (contours), heterogeneous medium 
Figure 11 shows brine content profiles at different distances from the drift at the end of test sequence $\mathrm{A}$. The profile intersecting the borehole (solid line) has reduced brine concentrations in the fracture zone as a result of formation water intrusion. Closer to the drift, higher brine contents near the fracture zone indicate preferential transport. These brine content profiles are almost symmetric with respect to the fracture. Only minor brine concentrations can be detected $0.2 \mathrm{~m}$ from the drift, restricted to the fracture zone. The front has not yet reached the drift wall.

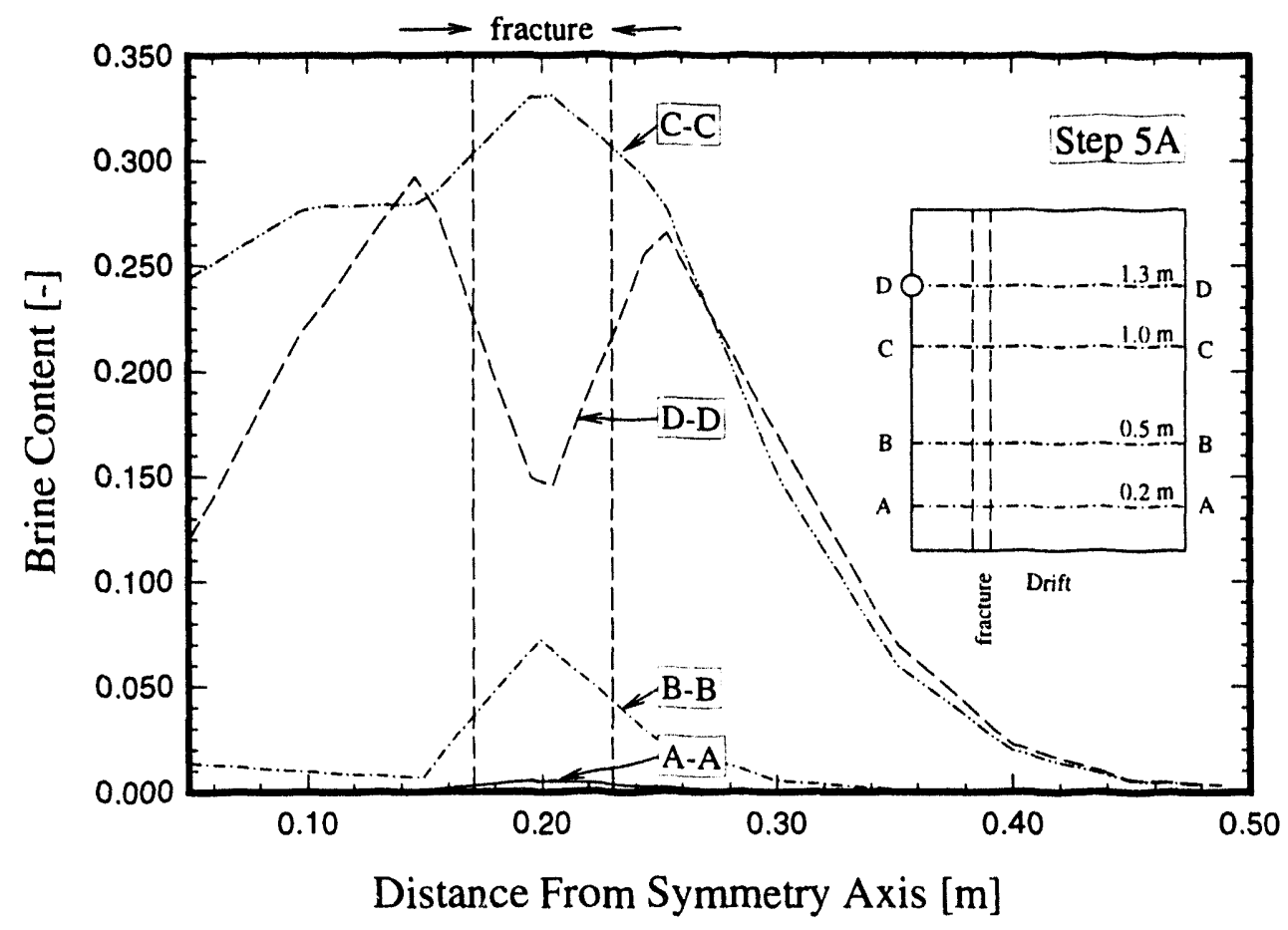

Figure 11: Brine content profiles at the end of test sequence $\mathrm{A}$

Profiles of brine content at the end of test sequence B are shown in Figure 12. Since less time has passed, the displacement of brine by fresh formation water is not as pronounced as at the end of test sequence A. Nevertheless, the preferential flow of liquid along the 
fracture zone leads to increased brine contents. Brine migrates towards the drift as a relatively narrow plume. In order to see brine concentrations, the sensors need to be placed close to this plume, the extent and location of which is a function of the mean flow direction and the dispersive processes.

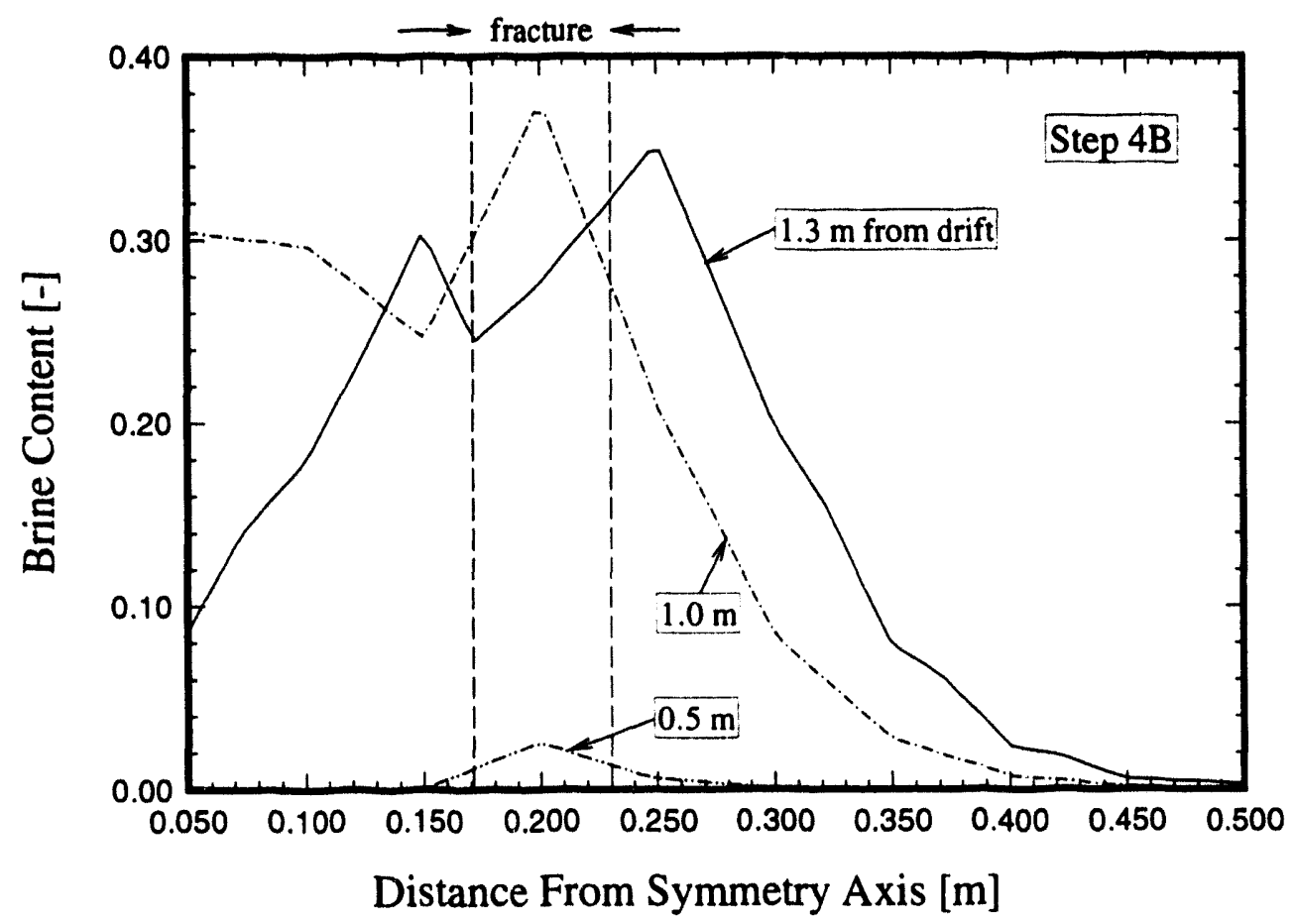

Figure 12: Brine content profiles at the end of test sequence B

Figure 13 shows brine contents predicted at four observation points. Since none of the sensors lies in the fracture zone, they respond rather slowly to the injection of brine. The strongest response can be seen at sensor TDR 1.90 which is closest to the injection borehole. However, after injection has stopped, brine flow rates are slow so that the maximum brine content is not observed within the simulated time period. Compared with the results of the homogeneous case, the TDR 1.60 sensor shows slightly lower brine contents indicating that a 
part of the saline solution bypasses the sensor. Sensors TDR1.30 and TDR1.10, which both are in a region of high gas saturation at a distance of $0.2 \mathrm{~m}$ from the fracture, do not show significant brine contents.

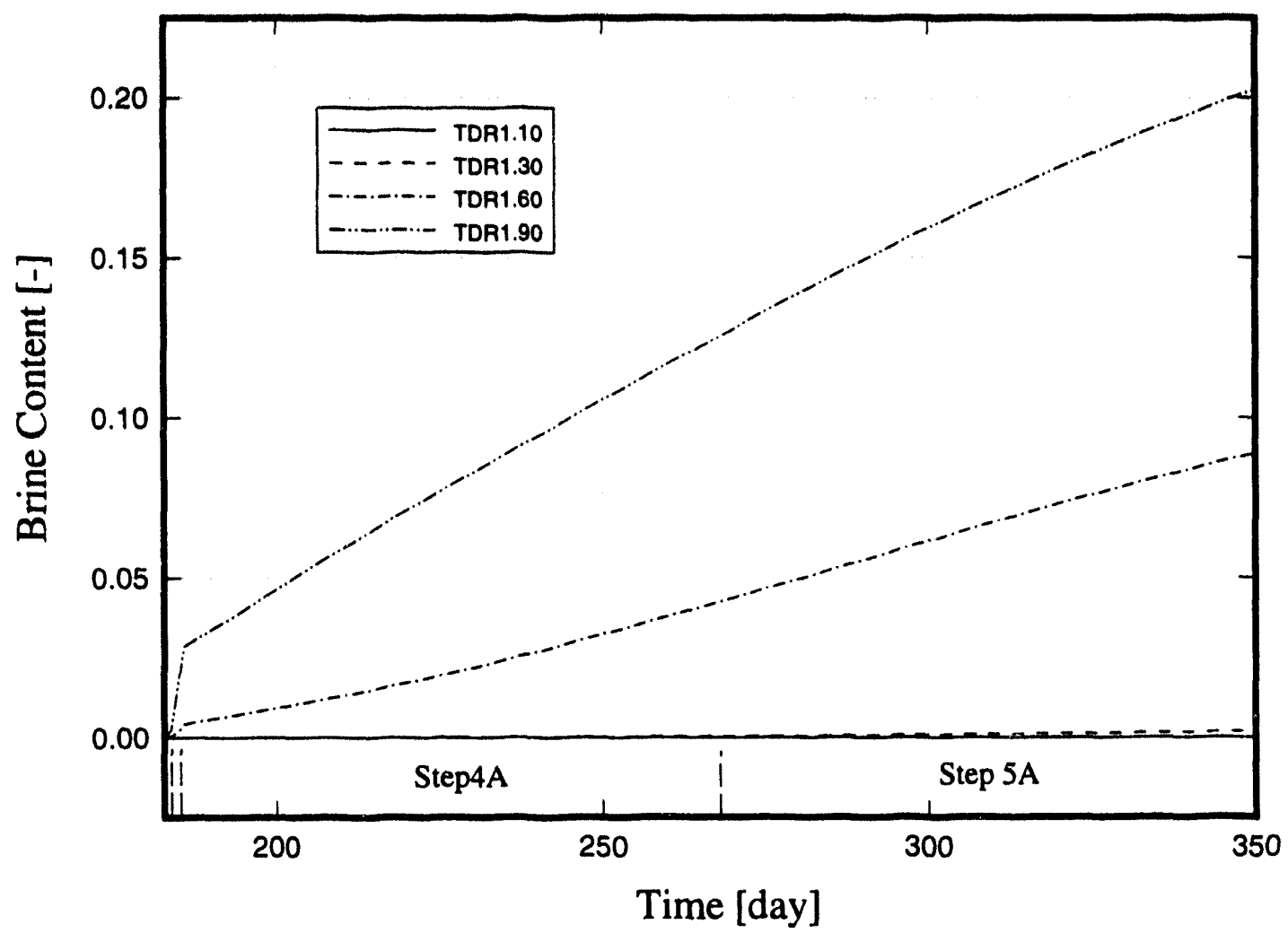

Figure 13: Brine content at TDR sensors, test sequence A, heterogeneous medium

It is interesting to note that no significant differences can be seen between Step 4A and Step 4B (Figures 13 and 14, respectively). Similar brine contents are observed at the TDR sensors regardless of the climatic condition in the drift. This can easily be explained by noting that the changed humidity is only affecting the sensors which are relatively close to the drift and which do not feel the effects of the brine plume yet. Conversely, the conditions at the deeper sensors which show brine contents are still very similar in both cases. 


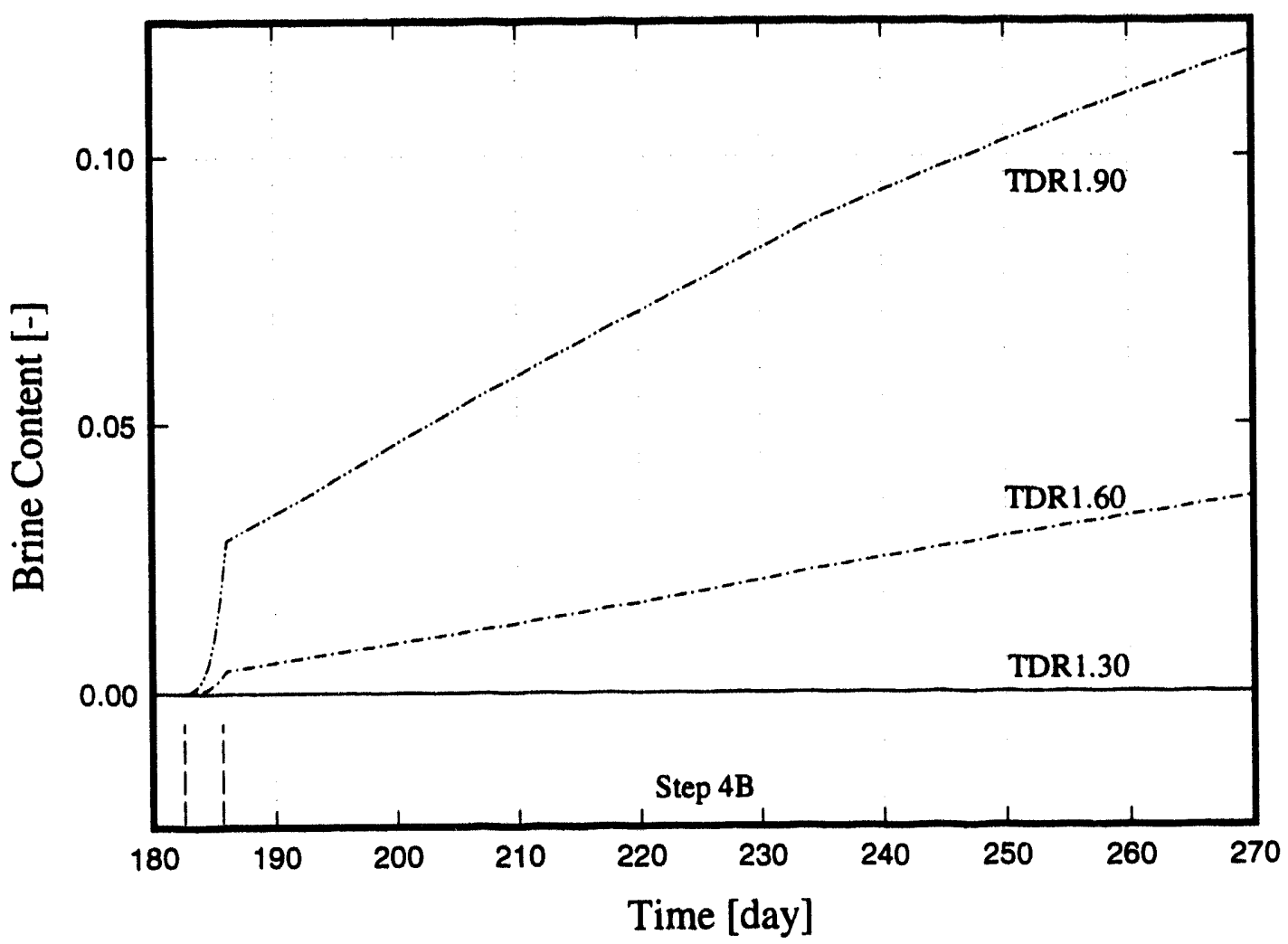

Figure 14: Brine content at TDR sensors, test sequence $B$, heterogeneous medium

Figures 15 and 16 show calculated water potentials for test sequences $\mathrm{A}$ and $\mathrm{B}$, respectively. Water potentials mainly reflect saturation conditions. Increased liquid saturation due to brine and water injection is only seen at sensor TP1.110 which is closest to the injection borehole. During resaturation (Step 5A or 4B), the two sensors TP1.2 and TP1.5 react much faster than those which are deeper in the formation. They also exhibit higher water potentials indicating that the pore space is refilled from the drift wall by vapor condensation rather than from the formation by Darcy flow of liquid water. 


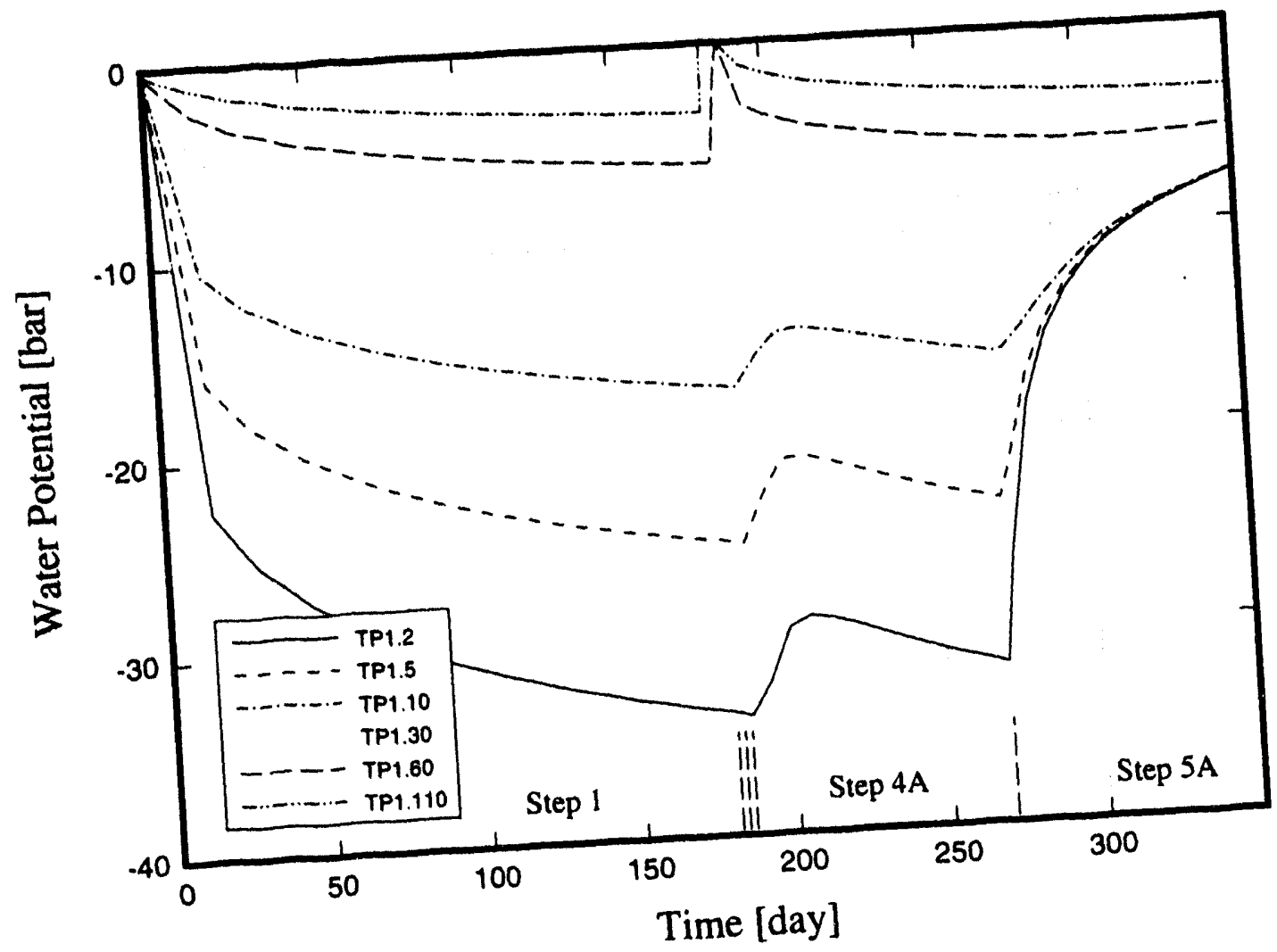

Figure 15: Water potential at TP sensors, test sequence A, heterogeneous medium

During resaturation (Step 5A in Figure 15, and Step 4B in Figure 16), water potential increases rapidly at sensors which are close to the drift. At the same time, the two deepest psychrometers show decreasing water potentials which are due to the redistribution of water driven by capillary pressure gradients. 


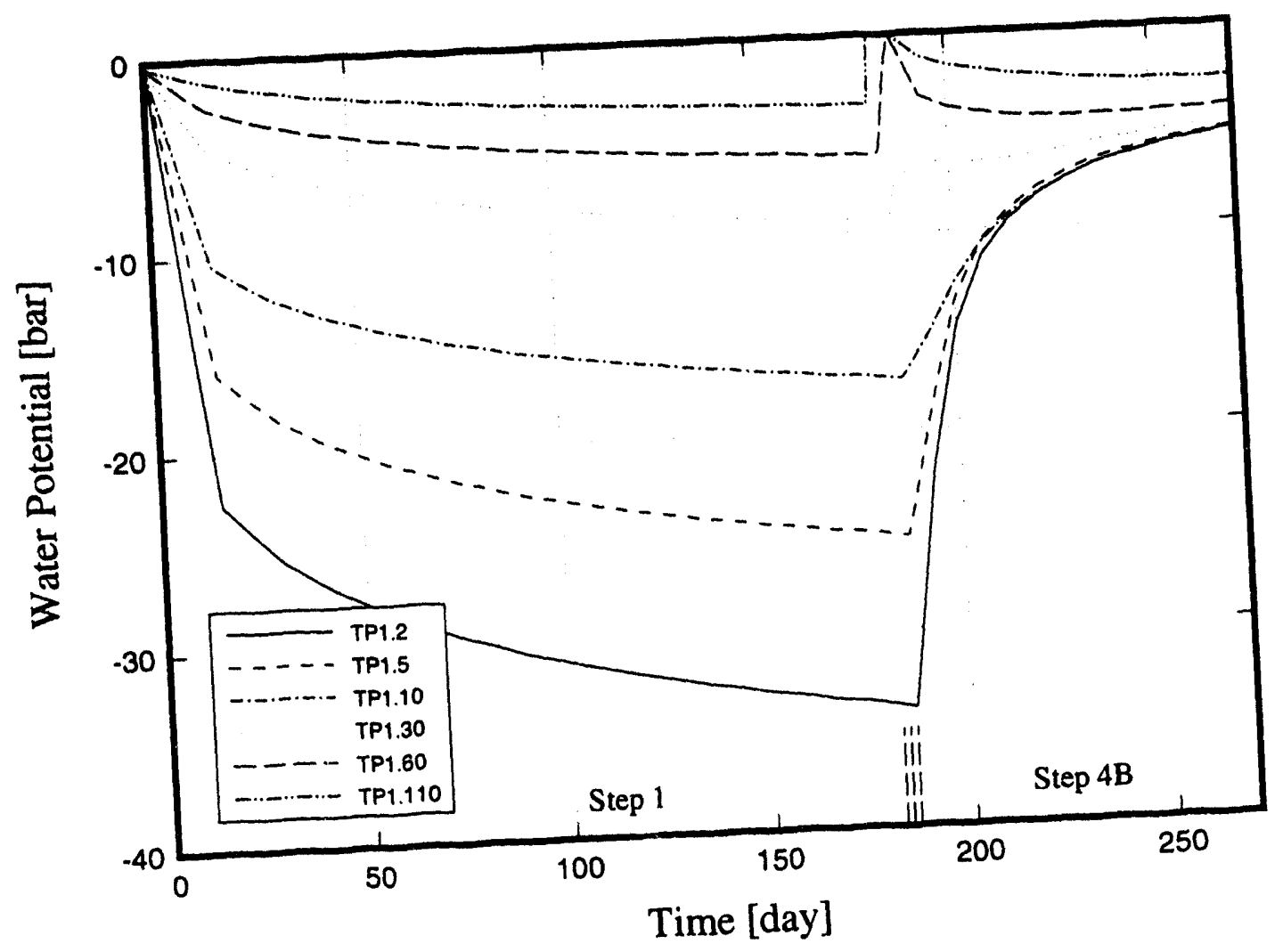

Figure 16: Water potential at TP sensors, test sequence $B$, heterogeneous medium 


\section{Discussion and Concluding Remarks}

The combined ventilation and brine injection experiments conceived to study liquid and gas transport near a drift with significant air circulation produce a variety of complicated interactions among the different fluids. The processes triggered by injecting brine and fresh water in borehole BOVE 84.011 may affect the transport of the trace electrolyte. Consequently, the velocities derived from electric conductivity and water potential measurements at different locations have to be carefully interpreted, taking into account the changed phase conditions in the vicinity of the borehole, which greatly affect the capillary pressure gradient, as one of the main driving forces.

It has been shown by our simulations that brine transport is a very slow process even under resaturating conditions. The full transition from gas-water to saline water and eventually fresh water cannot be observed within the given time frame even at sensors which are relatively close to the injection borehole.

Heterogeneity may locally accelerate the propagation of the different phases. Preferential flow of brine either enhances or reduces the salt concentrations at the observation points, depending on the location of the sensor with respect to the drift, the fracture zone, and the borehole. Furthermore, regions of weak capillary pressure may be drained leading to a flow barrier for liquid water and brine. The effect of increased permeability and reduced liquid saturation in the fracture zone are counteracting under steady-state flow conditions. As a result, the impact of the heterogeneity on the predicted brine content and water potential measurements is not very strong for the given parameter values. Preferential flow in a twophase flow system depends on the actual permeability and capillary pressure ratio between the fracture zone and the matrix. Furthermore, transient effects such as locally increased liquid saturations during the injection period are of great importance. They lead to increased 
liquid flow along the fracture zone in the vicinity of the borehole. Further downstream, flow in the fracture zone is reduced due to increased gas saturations.

Two test sequences have been proposed. If the propagation of the brine plume is of primary interest, test sequence B with a prolonged final test step is recommended. Test sequence A exhibits an additional cycle of drying and rewetting which may be of interest when studying hysteresis. The impact of test Step $4 \mathrm{~A}$ on the brine transport is very minor. The radial geometry of the flow field in combination with dispersion processes may lead to very low salinity values at the observation points. However, it is believed that the sensitivity of thi TDR equipment may be sufficient to detect changes of the bulk electric conductivity [Malicki et al., 1992].

The results of the simulations presented here should be considered preliminary. Potentially significant effects such as hysteresis and vapor pressure lowering in the formation have not been modeled. The vapor transfer at the drift wall is an important mechanism controlling evaporation rates which deserves further investigation. In the numerical model, a very simplified approach has been chosen to simulate moisture transfer at the drift wall. This may lead to an overestimation of evaporation rates and to an unrealistic saturation distribution near the drift. As shown in this study, the explicit modeling of heterogeneity is essential for understanding the potential system behavior. Finally, parameter uncertainty leads to relatively large prediction errors even if it is assumed that the underlying conceptual model is correct.

As a result of the large model and parameter uncertainties, these design célculations have been used to better understand the physical processes which influence the experiment rather than to predict the actual response at each of the sensors. On the other hand, the preliminary inverse modeling effort presented in this report shows that the system behavior can be accurately reproduced by numerical simulations. More sophisticated process models are available in recent TOUGH 2 enhancements to better represent the physics of saline water 
flow under unsaturated conditions, including brine dispersion, salt precipitation, and vapor pressure lowering due to capillary suction. Gravity may be added to simulate buoyancy effects.

Finally, after successful completion of the field tests, a careful interpretation of the experimental results using the TOUGH2 code will help elucidate the physical processes of multi-phase multi-component transport active in ventilation tests.

\section{Acknowledgment}

This work was supported, through U.S. Department of Energy Contract No. DE-AC0376SF00098, by the Director, Office of Civilian Radioactive Waste Management, Office of External Relations, administered by the Nevada Operations Office in cooperation with the Swiss National Cooperative for the Disposal of Radioactive Waste (Nagra). We would like to thank Th. Gimmi, Institute of Terrestrial Ecology, ETH Zürich, for a useful discussion of the planned experiment and for providing most of the input data. The review of this report by S.

Vomvoris and B. Frieg (Nagra), C. Oldenburg, G. Bodvarsson, and J. Long (LBL) is greatly appreciated. 


\section{References}

Cary, J. W. and S. A. Taylor, The interaction of the simultaneous diffusions of heat and water vapor, Soil Sci. Soc. Am. Proc., 26, 413-416, 1962a.

Cary, J. W. and S. A. Taylor, Thermally driven liquid and vapor phase transfer of water and energy in soil, Soil Sci. Soc. Am. Proc., 26, 417-420, 1962b.

Connell, L. D., P. R. F. Bell, Modeling moisture movement in revegetating waste heaps, 1. Development of a finite element model for liquid and vapor transport, Water Resou. Res. 29(5), 1435-1443, 1993.

Edlefsen, N. E. and A. B. C. Anderson, Thermodynamics of soil moisture, Hilgardia, 15(2), 31-298, 1943.

Finsterle, S., S. Vomvoris, Inflow to Stripa Validation Drift under two-phase conditions: Scoping calculations, Nagra Internal Report, Wettingen, Switzerland, 1991.

Finsterle, S., ITOUGH2 User's Guide, Lawrence Berkeley Laboratory Report LBL-34581, Berkeley, CA , 1993b.

Gimmi, T., H. Wydler, T. Baer, H. Abplanalp, H. Fluehler, Near field desaturation experiment at the Grimsel Test Site (FLG), Nagra Internal Report, Wettingen, Switzerland, 1992.

Hanks, R. J., H. R. Gardner and M. L. Fairbourn, Evaporation of water from soils as influenced by drying with wind or radiation, Soil Sci. Soc. Am. Proc., 31, 593-598, 1967.

Jury, W. A. and J. Letey, Jr., Water vapor movement in soil: Reconciliation of theory and experiment, Jour. Soil Sci. Am., 43(5), 823-827, 1979.

Malicki, M., R. Walczak, H. Wydler, T. Gimmi, H. Fluehler, Monitoring movement of a moisture and salt front in granite using the CAMI-TDR equipment, Nagra Internal Report, Wettingen, Switzerland, 1992. 
Oldenburg, C., K. Pruess, A two-dimensional dispersion module for the TOUGH2 simulator, Lawrence Berkeley Laboratory Report LBL-32505, Berkeley, CA, 1993.

Pruess, K, TOUGH2 - A general-purpose numerical simulator for multiphase fluid and heat flow, Lawrence Berkeley Laboratory Report LBL-29400, Berkeley, CA, 1991a.

Philip, J. R. and D. A. de Vries, Moisture movement in porous materials under temperature gradients, Tran. Am. Geophys. Union, 38, 222-231, 1957.

Pruess, K., EOS7, An equation-of-state module for the TOUGH2 simulator for two-Phase flow of saline water and air, Lawrence Berkeley Laboratory Report LBL-31114, Berkeley, CA, $1991 \mathrm{~b}$.

Ross, B., A conceptual model of deep unsaturated zones with neglibible recharge, Water Resou. Res. 20(11), 1627-1629, 1984.

Schneebeli, M., H.P. Laeser, H. Wydler, H. Fluehler, Wasserpotential und Wassergehalt in ungesättigten Graniten des Felslabors Grimsel: Labormessungen, Nagra Interner Bericht, Wettingen, Switzerland, 1990.

Schneebeli, M., T. Baer, H. Abplanalp, H. Wydler, H.P. Laeser, H. Fluehler, Water content and water potential in unsaturated granite: experiment, Nagra Internal Report, Wettingen, Switzerland, 1992.

Thiem, G., Hydrologische Methoden, Gebhardt, Leipzig, 1906.

Tsang, Y.W., K. Pruess, Further modeling studies of gas movement and moisture Mmigration at Yucca Mountain, Nevada, Lawrence Berkeley Laboratory Report LBL29127, Berkeley, CA, 1990.

Weeks, L. V., S. J. Richards and J. Letey, Water and salt transfer in soil resulting from thermal gradients, Soil Sci. Soc. Am. Proc., 32, 193-197, 1968. 
Vomvoris, S., B. Frieg (eds), Grimsel Test Site, Overview of Nagra field and modeling activities in the Ventilation Drift (1988 - 1990), Nagra Technical Report NTB 91-34, Wettingen, Switzerland, 1991.

Watanabe, K., Evaporation measurement in the Validation Drift - Part 1, Stripa Technical Report, TR 91-06, 1991.

Wilson, C. R., J. C. S. Long, R. M. Galbraith, K. Karasaki, H. K. Endo, H. O. Du Bois, Mc Pherson and G. Ramquist, Geohydrological data from the macro-permeability experiment at Stripa, Sweden, Lawrence Berkeley Laboratory Report LBL-12520, Berkeley, CA, 1981. 

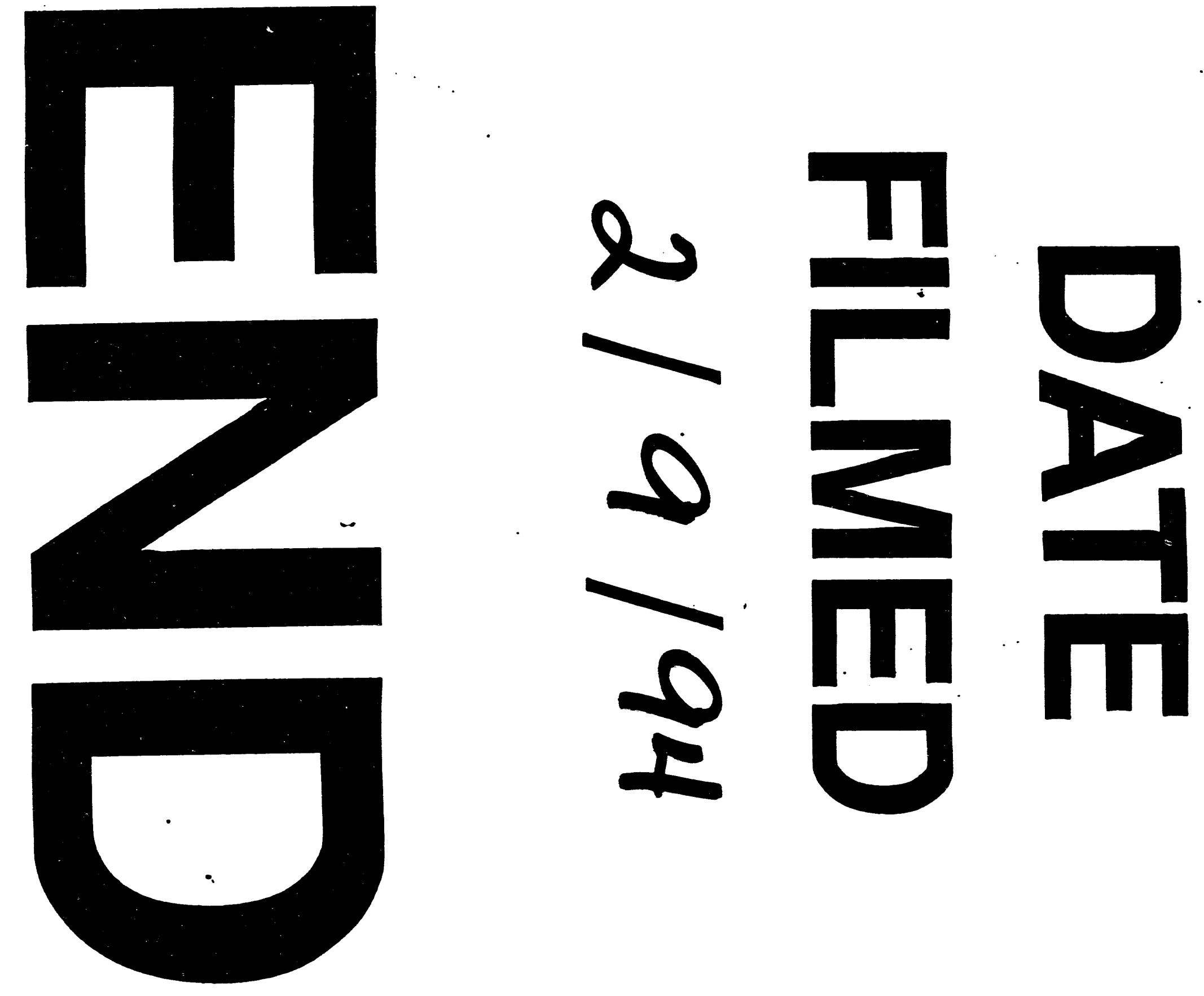
